Pure and Applied Mathematics Quarterly

Volume 7, Number 4

(Special Issue:

In memory of Eckart Viehweg)

1599-1629, 2011

\title{
A Characterization of Special Subvarieties in Orthogonal Shimura Varieties
}

\author{
Stefan Müller-Stach and Kang Zuo
}

Für Eckart, in Freundschaft und Dankbarkeit

\begin{abstract}
Let $A_{g}$ be the moduli space of principally polarized abelian varieties of dimension $g$ with some level structure and $M^{0} \subset A_{g}$ an orthogonal Shimura variety. We consider a smooth toroidal compactification $M$ of $M^{0}$ and a subvariety $Y \subset M$ intersecting the boundary of $M$ transversely. Then we give necessary and sufficient conditions of André-Oort type for $Y$ itself being the compactification of a special subvariety $Y^{0} \subset M^{0}$.
\end{abstract}

Keywords: André-Oort conjecture, Shimura variety, local system, Higgs bundle.

\section{INTRODUCTION}

In this paper we want to study a certain weak form of the André-Oort conjecture extending our previous work with Viehweg [19]. In order to explain our results we first want to introduce the required notation.

Received: Sept. 14, 2010; Revised: Jan. 24, 2011

1991 Mathematics Subject Classification. 14G35.

Supported by SFB/TRR 45 der Deutschen Forschungsgemeinschaft 2007-2011, Fudan University 2007 
Notation. Let $A_{g}:=A_{g}^{[N]}$ denote a fine moduli scheme of principally polarized abelian varieties of dimension $g$ with a level $N$-structure, for some $N \geq 3$. We choose in addition a smooth toroidal compactification $\bar{A}_{g}$ as constructed by Mumford et al. [1, chap. III], such that $S=\partial \bar{A}_{g}$ is a divisor with normal crossings. We denote by $f: X \rightarrow A_{g}$ the universal family of abelian varieties and by $\mathbb{V}=R^{1} f_{*} \mathbb{Q}$ the local system attached to it. There is a polarized variation of Hodge structures (VHS) defined over $\mathbb{Q}$ with underlying local system $\mathbb{V}$ which we also denote by $\mathbb{V}$. The assumption $N \geq 3$ implies that the monodromies of $\mathbb{V}$ around all components of $S$ are unipotent. We consider a smooth projective subvariety $Y \subset \bar{A}_{g}$ meeting $S$ transversely and define $Y^{0}:=Y \cap A_{g}$. Throughout this paper we denote subvarieties contained in the locally symmetric part $A_{g}$ of $\bar{A}_{g}$ with a superscript 0 .

Write $\left(G S p_{2 g}, H_{g}\right)$ for the pure Shimura datum defining $A_{g}=A_{g}^{[N]}$ with level structure given by the compact open subgroup $K(N)$ of $G S p_{2 g}\left(\mathbb{A}_{f}\right)$. By special subvariety of $A_{g}$ we mean, as is defined in [10] and [18, 6.2], a geometrically irreducible component of a Hecke translate of the image of some morphism $S h_{K}(G, X) \rightarrow A_{g}=S h_{K(N)}\left(G S p_{2 g}, H_{g}\right)$, which is defined by an inclusion of Shimura subdatum $(G, X) \subset\left(G S p_{2 g}, H_{g}\right)$ together with some compact open subgroup $K \subset G\left(\mathbb{A}_{f}\right)$ such that $K \subset K(N)$. More concretely, we abuse the notation $A_{g}=\Gamma(N) \backslash H_{g}^{+}$for a fixed connected component of $S h_{K(N)}\left(G S p_{2 g}, X\right)$, and we mainly work with subvarieties of $A_{g}$ that are of the form $\Gamma \backslash X^{+}$, where for some Shimura subdatum $(G, X)$ one has $X^{+}$a connected component of $X$, and $\Gamma=G(\mathbb{Q})_{+} \cap \Gamma(N)$. Note that the center of $G(\mathbb{R})$ acts on $X$ trivially, and $X^{+}$is homogeneous under $G^{a d}(\mathbb{R})^{+}$.

Typical cases of special subvarieties are given by moduli subschemes of $A_{g}$ that classify abelian varieties with PEL data. We refer the readers to [11, Section $4,5]$ and [14, Section 8, 8.14, 8.15, 8.17, etc.] for more details. Following the notations in [14], the subdatum $(G, X) \subset\left(G S p_{2 g}, H_{g}\right)$ can be given as follows. Consider $B$ a simple $\mathbb{Q}$-algebra endowed with a positive anti-involution $*$, and $(V, \psi)$ a symplectic $(B, *)$-module. Let $G$ be the linear $\mathbb{Q}$-group of $B$-linear symplectic similitudes of $V$. The following moduli problem of tuples $\left(A, s, i, \eta_{K}\right)$ is representable:

(i) $A$ is a complex abelian variety, with $\pm s$ a polarization of the Hodge structure $H_{1}(A, \mathbb{Q})$; 
(ii) $i$ a homomorphism $B \rightarrow \operatorname{End}(A) \otimes_{\mathbb{Q}} \mathbb{A}_{f}, \eta: V \otimes_{\mathbb{Q}} \mathbb{A}_{f} \simeq H^{1}(A, \mathbb{Q}) \otimes_{\mathbb{Q}} \mathbb{A}_{f}$ a $B \otimes_{\mathbb{Q}} \mathbb{A}_{f}$-linear isomorphism sending $\psi$ to an $\mathbb{A}_{f}^{\times}$-multiple of $s$, and $\eta_{K}$ is a $K$-orbit of $\eta, K$ being some compact open subgroup of $G\left(\mathbb{A}_{f}\right)$, which is assumed to be sufficiently small so as to preserve a level $N$ structure on $H_{1}(A, \mathbb{Q})$;

(iii) there exists a $B$-linear isomorphism $a: H_{1}(A, \mathbb{Q}) \rightarrow V$ sending $s$ to a $\mathbb{Q}^{\times}$multiple of $\psi$.

The moduli problem is represented by a Shimura variety $S h_{K}(G, X)$, with its canonical map into $A_{g}$.

In order to obtain special subvarieties of unitary type, one may take $B$ to be a central simple $E$-algebra, with $E$ some $C M$ extension of a totally real number field $F$, such that the restriction of $*$ to $E$ gives the complex conjugation fixing $F$. In particular, if one takes $V=B$ as a $\mathbb{Q}$-vector space, with $\psi$ given by some some element $q \in B^{\times}$such that $\psi(x, y)=\operatorname{tr}_{B / \mathbb{Q}}\left(x q y^{*}\right)$ (e.g. $q$ is in $E$ such that $c(q)=-q)$. Then $G^{\text {der }}$ is a $\mathbb{Q}$-form of $\operatorname{Res}_{F / Q} S L_{m}$, with $m=\sqrt{\operatorname{dim}_{E} B}$, and $G(\mathbb{R})$ is a product of unitary groups, whose signatures depend on the signatures of $q$ along different embeddings $F \rightarrow \mathbb{R}$. In order to make $X^{+}$an Hermitian symmetric space associated to $S U(m-1,1)$, one should choose the data in such a way that $G(\mathbb{R})$ is the product of a unitary group of signature $(m-1,1)$ with other unitary groups of signature $(m, 0)$.

In $[9,10.2]$ a Shimura subdatum $(G \operatorname{Spin}(V), X) \subset\left(G S p\left(C^{+}(V), H\left(C^{+}(V)\right)\right)\right.$ of orthogonal type is constructed. Note that the special subvarieties obtained from this subdatum are of the form $\Gamma \backslash X^{+}$, with $X^{+}$the Hermitian symmetric domain associated to $S O(n-2,2)_{\mathbb{R}} \simeq(G \operatorname{Spin}(V))_{\mathbb{R}}^{a d}$. This reformulates the results in [5, Section 4, 5], which is inspired by [13], where Kuga and Satake constructed a morphism from the moduli variety of K3 surfaces to the Siegel moduli variety of abelian varieties (for $n=21$ ). Deligne's construction in [5] exactly fits into the formalism of Shimura data axiomized later in [6], except that it follows the traditional convention of signs for Hodge types.

On the special subvariety defined by $(G \operatorname{Spin}(V), X) \subset\left(G S p\left(C^{+}(V)\right), H\left(C^{+}(V)\right)\right)$ there exists a polarized $\mathbb{Q}$-VHS of type $\{(-2,0),(-1,-1),(0,-2)\}$, with Hodge numbers $h^{-2,0}=h^{0,-2}=1, h^{-1,-1}=n-2$. Recall that in [5, 4,5], from the Shimura datum $(S O(V) \simeq S O(n-2,2), D)$ and the natural representation of $\rho: S O(V) \rightarrow G L(V)$, one gets, for any $x \in D$, a polarized $\mathbb{Q}$-HS $(V, \rho \circ x)$ of type $\{(-1,1),(0,0),(1,-1)\}$ with Hodge numbers $h^{-1,1}=h^{1,-1}=1, h^{0,0}=n-2$. 
Note that in $\rho \circ x: \mathbb{S} \rightarrow G L\left(V_{\mathbb{R}}\right)$, the real multiplicative group $\mathbb{G}_{m} \subset \mathbb{S}$ acts trivially. Now lift $\rho$ to the natural representation $\rho^{\prime}: G \operatorname{Spin}(V) \rightarrow G L(V)$. Since the center of $G \operatorname{Spin}(V)$ conincides with that of $G L(V)$ and that $G \operatorname{Spin}(V)$ is the central extension of $S O(V)$ by $\mathbb{G}_{m}$, we deduce that for any $x \in X$ with respect to the Shimura datum $(G \operatorname{Spin}(V), X),\left(V, \rho^{\prime} \circ x\right)$ is a polarized $\mathbb{Q}$-HS with types and Hodge numbers prescribed as in the beginning of the paragraph, namely shifted from the ones in $[5]$ by $(-1,-1)$. Consequently, from $\rho^{\prime}$ one obtains a polarized $\mathbb{Q}$-VHS on the special subvariety defined by $(G \operatorname{Spin}(V), X)$, with the same Hodge numbers as $\left(V, \rho^{\prime} \circ x\right), \forall x \in X$.

Interested readers may also consider more general cases of indefinite quadratic spaces over a totally real number field, as studied in [12].

Let us explain some notation used in the statement of the following two theorems even if more details can be found in the subsequent sections. In this paper, the symbol $S_{Z}$ always denotes the divisor at infinity for any compactified subvariety $Z \subset \bar{A}_{g}$, i.e., the intersection $S_{Z}=Z \cap S$ where $S=\partial \bar{A}_{g}$ is the boundary of $\bar{A}_{g}$, whereas the "open" part $Z \backslash S_{Z}$ is denoted by $Z^{0}$. In all considerations and proofs in this paper we will always make the following general assumption: All divisors $S_{Z}=Z \cap S$ which arise from intersections of images of subvarieties $Z \subset \bar{A}_{g}$ with $S=\partial \bar{A}_{g}$ are divisors with normal crossings, i.e., the intersections are transverse. In particular we can speak about logarithmic differential forms on $Z$ with poles in $S_{Z}$. Many of our results may hold with weaker assumptions. However, we want to work out the principles here, and do not strive for maximal generality.

Let $Y \subset \bar{A}_{g}$ be a smooth subvariety and $W \subset Y$ a subvariety of $Y$ satisfying the above transversality as assumptions. We denote by

$$
N_{W / Y}=T_{Y}\left(-\log S_{Y}\right) / T_{W}\left(-\log S_{W}\right)
$$

the logarithmic normal bundle of $W$ in $Y$. Let $\rho$ be the Picard number of $Y$ and $\delta$ the number of crossings of $S \cap Y$. Let $i: M^{0} \hookrightarrow A_{g}$ be a special subvariety for the orthogonal group $S O(2, n)$, where $i$ is the so-called Kuga-Satake embedding [13], and such that its toroidal compactification $M$ also is embedded into $\bar{A}_{g}$. On $M^{0}$ there is a natural polarized VHS $\mathbb{V}^{\prime}$ of weight two and rank $n+2$ coming from the standard representation of $S O(2, n)$ in $G L(n+2)$. There is a natural proper inclusion $\mathbb{V}^{\prime} \subset i^{*} \mathbb{V}^{\otimes 2}$ as a polarized sub VHS which is explained for example in [5]. The local monodromy of $\mathbb{V}^{\prime}$ around $S_{M}$ is assumed to be unipotent as well. The (canonical) Deligne extension $\overline{\mathcal{V}}^{\prime}$ of $\mathcal{V}^{\prime}:=\mathbb{V}^{\prime} \otimes \mathcal{O}_{M^{0}}$ to $M$ carries a natural 
Hodge filtration, i.e., a descending filtration

$$
\mathcal{V}^{\prime}=F^{0} \supset F^{1} \supset \cdots
$$

by subvector bundles and a logarithmic Gauss-Manin connection $\bar{\nabla}: \overline{\mathcal{V}^{\prime}} \rightarrow \overline{\mathcal{V}^{\prime}} \otimes$ $\Omega_{M}^{1}\left(\log S_{M}\right)$ extending $\nabla: \mathcal{V}^{\prime} \rightarrow \mathcal{V}^{\prime} \otimes \Omega_{M}^{1}$. The graded object associated to this filtration together with the graded logarithmic Gauss-Manin connection $\vartheta$ is the corresponding logarithmic Higgs bundle $\left(E=E^{2,0} \oplus E^{1,1} \oplus E^{0,2}, \vartheta\right)$ under the Simpson correspondence [23, Main Theorem]. Note that only for VHS this correspondence is so simple. Griffiths' transversality for $\mathcal{V}^{\prime}$ translates into the fact that $\vartheta$ is given by maps

$$
\vartheta^{2,0}: E^{2,0} \longrightarrow E^{1,1} \otimes \Omega_{M}^{1}\left(\log S_{M}\right), \quad \vartheta^{1,1}: E^{1,1} \stackrel{\simeq}{\longrightarrow} E^{0,2} \otimes \Omega_{M}^{1}\left(\log S_{M}\right)
$$

and $\vartheta^{0,2}=0$. Integrability of $\nabla$ implies $\vartheta \wedge \vartheta=0$.

The Griffiths-Yukawa coupling $\vartheta_{Z}^{(2)}$ on a smooth subvariety $i: Z \hookrightarrow M$ intersecting $S_{M}$ transversely in $S_{Z}$ is defined as the composition

$$
\vartheta_{Z}^{(2)}:=\vartheta^{1,1} \circ \vartheta^{2,0}: i^{*} E^{2,0} \longrightarrow i^{*} E^{1,1} \otimes \Omega_{Z}^{1}\left(\log S_{Z}\right) \longrightarrow i^{*} E^{0,2} \otimes \Omega_{Z}^{1}\left(\log S_{Z}\right)^{\otimes 2} .
$$

Note that $\vartheta^{(2)}$ lands in $S^{2} \Omega_{Z}^{1}\left(\log S_{Z}\right)$ as the image in $i^{*} E^{0,2} \otimes \Omega_{Z}^{2}\left(\log S_{Z}\right)$ is zero by the condition $\vartheta \wedge \vartheta=0$.

In the following statements, the degree of a vector bundle $F$ with respect to a line bundle $L$ on a smooth projective variety $Y$ of dimension $d$ is defined as $\operatorname{deg}_{L}(F):=c_{1}(L)^{d-1} c_{1}(F)$. The slope is defined as $\mu_{L}(F):=\operatorname{deg}_{L}(F) / \operatorname{rank}(F)$.

Previous and new results. The André-Oort conjecture asserts that an irreducible subvariety $Y^{0} \subset A_{g}$ is special if and only if it contains a dense set of CM points. Klingler and Yafaev [10] have announced a proof of it using results of Ullmo and Yafaev and had to assume a generalized Riemann hypothesis.

Our methods in this paper are not very sensitive to CM points versus non-CM points. However, the André-Oort conjecture would also imply that the closure of any union of positive dimensional special subvarieties is again special.

Our main goal therefore is to prove the following consequence of the André-Oort conjecture: Let $Y^{0} \subset A_{g}$ be a subvariety containing sufficiently many special subvarieties of dimension $\geq 1$. Then $Y^{0}$ is special. 
The notion "sufficiently many" can be expressed for example in the following way. In [19, Thm. 4.4] we used special divisors $W_{i}^{0} \subset Y^{0}$ satisfying HirzebruchHöfer proportionality (HHP) with $i \in I$, a finite index set. Condition (HHP) is an equality condition arising from an inequality which in turn has its origin in natural stability conditions for Higgs bundles. We refer to [19] for the history of this condition.

In [19] we then showed that $Y^{0}$ is special if $\sharp I$ exceeds some effective bound:

Theorem (Thm. 4.4 in [19]). Let $Y \subset M \subset \bar{A}_{g}$ be a subvariety of $\bar{A}_{g}$ contained in a toroidal compactification $M$ of a Shimura subvariety $M^{0} \subset A_{g}$ of type $S O(2, d)$. We assume that $Y$ and $M$ intersect the boundary $S$ of $A_{g}$ transversely, and require that $\Omega_{Y}^{1}\left(\log S_{Y}\right)$ is nef and $\omega_{Y}\left(S_{Y}\right)$ is ample with respect to $Y^{0}$. Assume $\operatorname{dim}(Y) \geq 2$ and $W_{i} \subset Y \quad(i \in I=$ finite set) are pairwise distinct divisors such that $W_{i}^{0} \subset M^{0}$ is special.

(i) If all $W_{i}^{0}$ are of orthogonal type, if all $W_{i}$ satisfy condition

$$
(H H P): \quad \frac{\operatorname{deg}_{\omega_{W_{i}}\left(S_{W_{i}}\right)}\left(N_{W_{i} / Y}\right)}{\operatorname{rank} N_{W_{i} / Y}}=\frac{\operatorname{deg}_{\omega_{W_{i}}\left(S_{W_{i}}\right)}\left(T_{W_{i}}\left(-\log S_{W_{i}}\right)\right)}{\operatorname{rank} T_{W_{i}}\left(-\log S_{W_{i}}\right)},
$$

and if $\# I \geq \varsigma(Y):=(\rho+\delta)^{2}+\rho+\delta+1$, then $Y^{0} \subset M^{0}$ is a special subvariety of orthogonal type.

(ii) Assume that the Griffiths-Yukawa coupling vanishes on $Y$. If the $W_{i}^{0}$ are special subvarieties of unitary type, if condition (HHP) holds

$$
(H H P): \quad \frac{\operatorname{deg}_{\omega_{W_{i}}\left(S_{W_{i}}\right)}\left(N_{W_{i} / Y}\right)}{\operatorname{rank} N_{W_{i} / Y}}=\frac{\operatorname{deg}_{\omega_{W_{i}}\left(S_{W_{i}}\right)}\left(T_{W_{i}}\left(-\log S_{W_{i}}\right)\right)}{d+1},
$$

and if $\# I \geq \varsigma(Y)$, then $Y^{0} \subset M^{0}$ is a special subvariety of unitary type.

(iii) Let $Y$ be a surface and $I=\{1,2\}$. Assume that

$$
\sigma_{1}\left(W_{1}\right) \cap \sigma_{2}\left(W_{2}\right) \neq \emptyset
$$

and $\operatorname{deg} N_{W_{i} / Y}=0$ for $i=1,2$. Then $Y^{0}$ is the product of two Shimura curves.

In the following main result in this paper we remove the divisor hypothesis and obtain necessary and sufficient conditions supporting the André-Oort conjecture.

We need some additional notation to explain the theorem: We say that $Y$ can be covered by a smoothing of a cycle $\sum_{i} a_{i} C_{i}$ of compactified special curves 
$C_{i}^{0} \subset Y^{0}$ satisfying (HHP), if there are finitely many embedded special curves $C_{i}^{0} \subset Y^{0}$ satisfying (HHP) such that their compactifications $C_{i} \subset Y$ admit a linear combination $\sum_{i} a_{i} C_{i}$ with integer coefficients which can be deformed as embedded cuves in $Y$ in a family, such that the general deformation is smooth. Condition (HHP) in this case is given by equality in the following inequality:

$$
\operatorname{deg} N_{C / Y} \leq \frac{\operatorname{rank}\left(N_{C / Y}^{1}\right)+\operatorname{rank}\left(N_{C / Y}^{0}\right)}{2} \cdot \operatorname{deg} T_{C}\left(-\log S_{C}\right)
$$

Here $N_{C / Y}^{\bullet}$ is the Harder-Narasimhan filtration on the logarithmic normal bundle $N_{C / A_{g}}$ intersected with $N_{C / Y}$. We also fix $C_{1}$ and a base point $y_{0} \in C_{1}$. With the notation for the Higgs bundle $E$ on $M$ restricted to $Y$ we then define the following vector spaces: $W_{y_{0} \in Y}$ is the subspace of vectors in $E_{y_{0}}^{1,1}$ vanishing unter $\vartheta$ at the base point $y_{0}$ and $W_{y_{0} \in Y, \mathbb{R}} \subset W_{y_{0} \in Y}$ the real subspace of real vectors in $W_{y_{0} \in Y}$.

Theorem 3.6. Let $Y \subset M \subset \bar{A}_{g}$ be a subvariety of $\bar{A}_{g}$ contained in a toroidal compactification $M$ of a Shimura subvariety $M^{0} \subset A_{g}$ of type $S O(2, n)$. We assume that $Y$ and $M$ intersect the boundary $S$ of $A_{g}$ transversely, and that $Y$ can be covered by a smoothing of a cycle $\sum_{i} a_{i} C_{i}$ of compactified special curves $C_{i}^{0} \subset Y^{0}$ satisfying $(H H P)$. Then:

(a) If $W_{y_{0} \in Y}=W_{y_{0} \in Y, \mathbb{R}} \otimes \mathbb{C}$ for some $y_{0} \in C_{1}$ then $Y^{0} \subset M^{0}$ is a special subvariety of orthogonal type.

(b) If the Griffiths-Yukawa couplings along all $C_{i}$ do not vanish then $Y^{0} \subset M^{0}$ is a special subvariety of orthogonal type.

(c) If the Griffiths-Yukawa coupling along $Y$ vanishes then $Y^{0} \subset M^{0}$ is a special subvariety of unitary type, i.e., a ball quotient.

In the assertions (a) and (b) of this theorem one may replace the assumption on the smoothing of the cycle $\sum_{i} a_{i} C_{i}$ of special curves $C_{i} \subset Y$ satisfying (HHP) by the following: Assume that there is a connected union $C_{1} \cup \cdots \cup C_{l}$ of compactified special curves satisfying (HHP) and such that the image $\pi_{1}\left(\bigcup C_{i}^{0}, *\right)$ under the natural map in $\pi_{1}\left(Y^{0}, *\right)$ is big in the sense that the image of $\pi_{1}\left(\bigcup C_{i}^{0}, *\right)$ under the monodromy representation restricted to $Y^{0}$

$$
\pi_{1}\left(\bigcup C_{i}^{0}, *\right) \longrightarrow \pi_{1}\left(Y^{0}, *\right) \stackrel{\rho}{\rightarrow} S O(2, n)
$$


is Zariski dense in the algebraic monodromy group $H\left(Y^{0}\right)$, i.e., the $\mathbb{Q}$-algebraic closure of the monodromy representation $\rho$. We note that this is a subgroup of Hermitian type in $\mathrm{SO}(2, n)$. This is nicely explained, for example, in [17, Sect. 1.3]. Hermitian subgroups of $\mathrm{SO}(2, n)$ like $H\left(Y^{0}\right)$ can be classified. Besides the obvious orthogonal and unitary subgroups which are $\mathbb{Q}$-simple there are $\mathrm{SL}_{2} \times$ $\mathrm{SL}_{2}$ and quaternionic versions [21, Thm. 5.2.3.]. In the non- $\mathbb{Q}$-simple cases we therefore have $\operatorname{dim}(Y)=2$ and $Y^{0}$ is uniformized by a product $\mathbb{H} \times \mathbb{H}$ of upper half planes.

Acknowledgements. This work naturally continues the results in [19]. Together with Eckart Viehweg we have thought about thickenings of Higgs bundles during a stay at Fudan University in the summer of 2007. Thickenings play an essential role in this paper which therefore should be considered as joint work with Eckart.

We thank Ke Chen for explanations on Shimura data. We also thank Michael Harris for enlightening discussions about special cycles and the referee for several helpful remarks.

\section{BASIC SETUP}

In this section we will use the Simpson correspondence for curves [23, Main Thm.]. It is a natural equivalence between the category of direct sums of stable filtered regular Higgs bundles of degree zero and the category of direct sums of stable filtered local systems of degree zero. We will need this correspondence only in the case when the local system $\mathbb{V}$ has unipotent local monodromies. In that case the filtration on the Higgs bundle is trivial and $\operatorname{deg}(\mathbb{V})$ is automatically zero. We refer the reader to [25, sect. 1] for additional results and explanations on Higgs bundles on curves building up on Simpson's work.

Consider a non-singular projective curve $C$ and a non-constant morphism

$$
\varphi: C \rightarrow Y \subset \bar{A}_{g}
$$

where $Y \subset \bar{A}_{g}$ is a smooth projective subvariety as in the introduction. We set $C^{0}:=\varphi^{-1}\left(Y^{0}\right) \neq \emptyset$, where $Y^{0}=Y \cap A_{g}$ denotes the "open" part.

In the following we consider the situation where $C^{0}$ is a Shimura curve and $S_{C}:=C \backslash C^{0}$ is the set of cusps. We also denote by $S_{Y}$ the intersection of $Y$ with 
$S=\partial \bar{A}_{g}$ and we assume that the intersection is transversal such that $S_{Y}$ is a divisor with normal crossings. We assume that the restriction $\varphi: C^{0} \rightarrow Y^{0} \subset A_{g}$ is an étale morphism of Shimura varieties. Let $f: X \rightarrow C^{0}$ denote also the family obtained by pullback via $\varphi$.

The main goal of this paper is to find new criteria when $Y^{0}$ itself is a special subvariety in $A_{g}$, for example if "sufficently many" such curves $C$ with certain properties map to $Y$. In such a situation, by [25, Prop. 1.4] and [16, Thm. 0.9], after replacing $C^{0}$ by an étale cover, the local system $\mathbb{V}_{C^{0}}:=\varphi^{*} R^{1} f_{*} \mathbb{C}_{X}$ admits a decomposition

$$
\mathbb{V}_{C^{0}}=\mathbb{L} \otimes \mathbb{T} \oplus \mathbb{U}
$$

as a polarized complex variation in the sense of Deligne, i.e., a polarized $\mathbb{C}$-VHS in the sense of Simpson [23] on $C^{0}$. Note that this étale cover of $C^{0}$ is necessary, however, all our proofs below are insensitive to such étale base change even if we apply this construction to a finite number of curves simultaneously later. Here $\mathbb{L}$ is of weight one and rank two with the logarithmic Higgs bundle

$$
\left(\mathcal{L} \oplus \mathcal{L}^{-1}, \tau: \mathcal{L} \simeq \mathcal{L}^{-1} \otimes \Omega_{C}^{1}\left(\log S_{C}\right)\right)
$$

$\mathbb{T}$ is concentrated in bidegree $(0,0)$ and selfdual, whereas $\mathbb{U}$ is of weight one and decomposes in two local subsystems

$$
\mathbb{U}=\mathbb{U}^{1,0} \oplus \mathbb{U}^{0,1}, \quad \mathbb{U}^{1,0}=\mathbb{U}^{0,1 \vee} .
$$

Note that the local systems $\mathbb{T}, \mathbb{U}^{1,0}$ and $\mathbb{U}^{0,1}$ are unitary and the local monodromies around $S_{C}$ are unipotent, hence the local monodromies are in fact trivial. Hence $\mathbb{T}, \mathbb{U}^{1,0}$ and $\mathbb{U}^{0,1}$ can be extended as local systems to $C$. Writing $\left(\mathcal{T}=\mathbb{T} \otimes \mathcal{O}_{C}, 0\right)$ and $\left(\mathcal{U}=\mathbb{U}^{1,0} \otimes \mathcal{O}_{C}, 0\right) \oplus\left(\mathcal{U}^{\vee}=\mathbb{U}^{0,1} \otimes \mathcal{O}_{C}, 0\right)$ for the corresponding Higgs bundles, then the Higgs bundle corresponding to $\mathbb{V}_{C^{0}}$ decomposes in the form

$$
\left(E^{1,0} \oplus E^{0,1}, \theta\right)=\left(\mathcal{L} \oplus \mathcal{L}^{-1}, \tau\right) \otimes(\mathcal{T}, 0) \oplus(\mathcal{U}, 0) \oplus\left(\mathcal{U}^{\vee}, 0\right) .
$$

The line bundle $\mathcal{L}$ has positive degree, since $\mathcal{L}$ is the pullback of some positive power of the automorphic line bundle on $\bar{A}_{g}$ via $\varphi: C \rightarrow \bar{A}_{g}$. Since $\varphi: C^{0} \rightarrow A_{g}$ is not constant and the automorphic line bundle is positive on $\bar{A}_{g}$ it follows that $\operatorname{deg}(\mathcal{L})$ is positive. Via the isomorphism $\tau$ we identify $T_{C}\left(-\log S_{C}\right)=\mathcal{L}^{-2}$. 
The Hodge metric $\mathbb{V}_{C^{0}}$ comes from the tensor product of the Hodge metrics on $\mathbb{L}, \mathbb{T}, \mathbb{U}^{1,0}$ and $\mathbb{U}^{0,1}$, which, by [24, Sect. 4] and [23], coincide with the HermitianYang-Mills metrics on the corresponding logarithmic Higgs bundles. The Hodge metrics on $E^{1,0}$ and $E^{0,1}$ are tensor products of the Hodge metrics on $\mathcal{L}^{ \pm}, \mathcal{T}, \mathcal{U}^{1,0}$ and $\mathcal{U}^{0,1}$.

In general, a Hodge bundle with Hodge metric of any Schur functor $S\left(\mathbb{V}_{C^{0}}\right)$ is obtained in a similar way from the Hodge metrics on $\mathcal{L}^{ \pm}, \mathcal{T}, \mathcal{U}^{1,0}$ and $\mathcal{U}^{0,1}$.

Let $f: X \rightarrow A_{g}$ denote the universal family, $\mathbb{V}:=R^{1} f_{*} \mathbb{C}_{X}$ and $E:=E^{1,0} \oplus E^{0,1}$ the logarithmic Higgs bundle corresponding to Deligne's canonical extension of $\mathbb{V} \otimes \mathcal{O}_{A_{g}}$ on the toroidal compactification $\bar{A}_{g} \supset A_{g}$. It comes with the logarithmic Higgs map

$$
\theta: E^{1,0} \rightarrow E^{0,1} \otimes \Omega \frac{1}{A_{g}}(\log S)
$$

Since $\mathbb{V}$ is a polarized VHS, there is a natural isomorphism $\operatorname{End}(E) \stackrel{\simeq}{\rightarrow} E^{\otimes 2}$. Then it is well-known [7, p. 339] that the composition

$$
T_{\bar{A}_{g}}(-\log S) \stackrel{\theta}{\rightarrow} \operatorname{End}(E) \stackrel{\simeq}{\rightarrow} E^{\otimes 2} \rightarrow S^{2}\left(E^{0,1}\right),
$$

identifies $T_{\bar{A}_{g}}(-\log S)$ with $S^{2}\left(E^{0,1}\right)$. The derivatives of the maps

$$
C \stackrel{\varphi}{\rightarrow} Y \stackrel{i}{\rightarrow} \bar{A}_{g}
$$

induce the following commutative diagramm

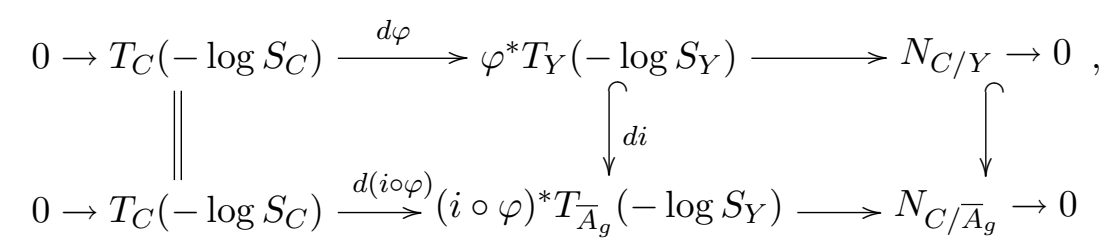

where $N_{C / Y}$ is the (logarithmic) normal bundle of $\varphi: C \rightarrow Y$ and $N_{C / \bar{A}_{g}}$ is the (logarithmic) normal bundle of $i \circ \varphi: C \rightarrow \bar{A}_{g}$.

On the curve $C$ one has

$(i \circ \varphi)^{*} T_{\bar{A}_{g}}(-\log S)=(i \circ \varphi)^{*} S^{2}\left(E^{0,1}\right)=\left(\mathcal{L}^{-2} \otimes S^{2}(\mathcal{T})\right) \oplus\left(\mathcal{L}^{-1} \otimes \mathcal{T} \otimes \mathcal{U}^{\vee}\right) \oplus S^{2}\left(\mathcal{U}^{\vee}\right)$ 
where the decomposition on the right side is induced by (1.1) and is orthogonal with respect to the Hodge metric.

All three summands are polystable by the main theorem in [23], but, as $\operatorname{deg}(\mathcal{L})>$ 0 , with different slopes

$$
-2 \operatorname{deg} \mathcal{L},-\operatorname{deg} \mathcal{L} \text { and } 0 .
$$

Consider the inclusion

$$
\begin{gathered}
T_{C}\left(-\log S_{C}\right) \stackrel{d \varphi}{\rightarrow} \varphi^{*} T_{Y}\left(-\log S_{Y}\right) \stackrel{d i}{\rightarrow}(i \circ \varphi)^{*} T_{\bar{A}_{g}}(-\log S)= \\
=\left(\mathcal{L}^{-2} \otimes S^{2}(\mathcal{T})\right) \oplus\left(\mathcal{L}^{-1} \otimes \mathcal{T} \otimes \mathcal{U}^{\vee}\right) \oplus S^{2}\left(\mathcal{U}^{\vee}\right),
\end{gathered}
$$

As the derivative $d \varphi$ can be identified with the Higgs map $\theta$ and $\theta$ on $C$ preserves the direct sum decomposition in (1.1) and vanishes on the second summand, the image of $T_{C}\left(-\log S_{C}\right)$ is contained in $\mathcal{L}^{-2} \otimes S^{2}(\mathcal{T})$.

For the convenience of the reader we recall the following definition.

Definition 1.1. A holomorphic subbundle $i: F \hookrightarrow E$ of a Hodge bundle $E$ of a polarized complex variation of Hodge structure is called a direct summand of $E$ and orthogonal with respect to the Hodge metric if there exists an isomorphism $E \simeq F \oplus G$ between holomorphic vector bundles, such that the first summand defines the inclusion $i$ and the decomposition is orthogonal with respect to the Hodge metric.

Lemma 1.2. The line subbundle

$$
T_{C}\left(-\log S_{C}\right) \subset \mathcal{L}^{-2} \otimes S^{2}(\mathcal{T})
$$

induces a holomorphic decomposition of $\mathcal{L}^{-2} \otimes S^{2}(\mathcal{T})$, which is orthogonal with respect the Hodge metric, i.e., there exists a holomorphic subbundle

$$
T_{C}\left(-\log S_{C}\right)^{\perp} \subset \mathcal{L}^{-2} \otimes S^{2}(\mathcal{T})
$$

such that

$$
\mathcal{L}^{-2} \otimes S^{2}(\mathcal{T})=T_{C}\left(-\log S_{C}\right) \oplus T_{C}\left(-\log S_{C}\right)^{\perp},
$$

and such that this decomposition is orthogonal with respect to the Hodge metric.

Proof. We note first that the Hodge metric on $\mathcal{L}^{-2} \otimes S^{2}(\mathcal{T})$ comes from the corresponding tensor product of the Hodge metrics on the polarized $\mathbb{C}$-VHS 
$\mathbb{L}, \mathbb{T}$ and $\mathbb{U}$. The Hodge metric on the corresponding Higgs bundle $\mathcal{T}$ is the Hermitian-Yang-Mills metric by [23]. Consider the subbundle

$$
T_{C}\left(-\log S_{C}\right) \subset \mathcal{L}^{-2} \otimes S^{2}(\mathcal{T}) .
$$

Since

$$
\tau: \mathcal{L} \simeq \mathcal{L}^{-1} \otimes \Omega_{C}^{1}\left(\log S_{C}\right),
$$

we have $T_{C}\left(-\log S_{C}\right)=\mathcal{L}^{-2}$, hence

$$
\mathcal{L}^{-\otimes 2} \subset \mathcal{L}^{-2} \otimes S^{2}(\mathcal{T}) .
$$

Dividing both sides by the factor $\mathcal{L}^{-\otimes 2}$ we get

$$
\mathcal{O}_{C} \subset S^{2}(\mathcal{T})
$$

Note that the Higgs bundle $S^{2}(\mathcal{T})$ has zero Higgs field. Hence $\mathcal{O}_{C}$ is a Higgs sub bundle of $S^{2}(\mathcal{T})$ with slope equality $\mu\left(\mathcal{O}_{C}\right)=0=\mu\left(S^{2}(\mathcal{T})\right)$. Applying Simpson's Higgs polystability, there exists a holomorphic decomposition

$$
S^{2}(\mathcal{T})=\mathcal{O}_{C} \oplus \mathcal{O}_{C}^{\perp}
$$

which is orthogonal w.r.t. the Hermitian-Yang-Mills metric on $S^{2}(\mathcal{T})$. Tensoring with $\mathcal{L}^{-\otimes 2}$ on both sides of the above decomposition, we obtain the desired decomposition as claimed.

The decomposition in Lemma 1.2 induces the following decompostion $(i \circ \varphi)^{*} T_{\bar{A}_{g}}\left(-\log S_{Y}\right)=T_{C}\left(-\log S_{C}\right) \oplus\left(T_{C}\left(-\log S_{C}\right)^{\perp} \oplus\left(\mathcal{L}^{-1} \otimes \mathcal{T} \otimes \mathcal{U}^{\vee}\right) \oplus S^{2}\left(\mathcal{U}^{\vee}\right)\right)$.

Let $p$ denote the projection to the first summand, then the composition

$$
T_{C}\left(-\log S_{C}\right) \stackrel{d \varphi}{\rightarrow} \varphi^{*} T_{Y}\left(-\log S_{Y}\right) \stackrel{d i}{\rightarrow}(i \circ \varphi)^{*} T_{\bar{A}_{g}}(-\log S) \stackrel{p}{\rightarrow} T_{C}\left(-\log S_{C}\right)
$$

is the identity. This shows that both horizontal short exact sequences in diagram (1.2) split in the form

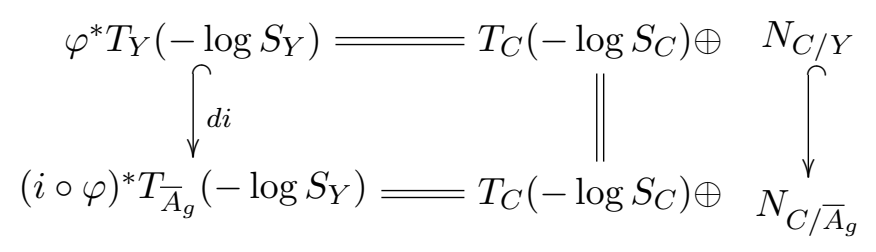


such that

$$
N_{C / \bar{A}_{g}}=T_{C}\left(-\log S_{C}\right)^{\perp} \oplus\left(\mathcal{L}^{-1} \otimes \mathcal{T} \otimes \mathcal{U}^{\vee}\right) \oplus S^{2}\left(\mathcal{U}^{\vee}\right) .
$$

Remark 1.3. The holomorphic and orthogonal splitting

$$
T_{C}(-\log S) \stackrel{d(i \circ \varphi)}{\longrightarrow}(i \circ \varphi)^{*} T_{\bar{A}_{g}}(-\log S)
$$

for a special curve $i \circ \varphi: C^{0} \rightarrow A_{g}$ in (1.3) holds also true in general if $C^{0}$ is replaced by any special subvariety, see the proof for ii) in Proposition 1.5. In diagram (1.3) we obtain an explicit description of the logarithmic normal bundle $N_{C / \bar{A}_{g}}$.

We shall now describe the Harder-Narasimhan filtration on $N_{C / \bar{A}_{g}}$. Let

$$
\begin{gathered}
N_{C / \bar{A}_{g}}^{0}:=T_{C}\left(-\log S_{C}\right)^{\perp}, \\
N_{C / \bar{A}_{g}}^{1}:=T_{C}\left(-\log S_{C}\right)^{\perp} \oplus \mathcal{L}^{-1} \otimes \mathcal{T} \otimes \mathcal{U}^{\vee}
\end{gathered}
$$

and

$$
N_{C / \bar{A}_{g}}^{2}:=N_{C / \bar{A}_{g}}
$$

Then the filtration

$$
0 \subset N_{C / \bar{A}_{g}}^{0} \subset N_{C / \bar{A}_{g}}^{1} \subset N_{C / \bar{A}_{g}}^{2}=N_{C / \bar{A}_{g}}
$$

is the Harder-Narasimhan filtration on $N_{C / \bar{A}_{g}}$. In our situation, the graded summands

$$
N_{C / \bar{A}_{g}}^{i} / N_{C / \bar{A}_{g}}^{i-1}, \text { for } 0 \leq i \leq 2
$$

are polystable vector bundles of slopes $\operatorname{deg} T_{C}\left(-\log S_{C}\right), \frac{1}{2} \operatorname{deg} T_{C}\left(-\log S_{C}\right)$, and 0 . One has

$$
\begin{aligned}
& N_{C / \bar{A}_{g}}^{1}=N_{C / \bar{A}_{g}}^{0} \oplus N_{C / \bar{A}_{g}}^{1} / N_{C / \bar{A}_{g}}^{0}, \\
& N_{C / \bar{A}_{g}}^{2}=N_{C / \bar{A}_{g}}^{1} \oplus N_{C / \bar{A}_{g}}^{2} / N_{C / \bar{A}_{g}}^{1} .
\end{aligned}
$$

Taking the induced filtration on $N_{C / Y} \subset N_{C / \bar{A}_{g}}$ obtained by intersection with $N_{C / \bar{A}_{g}}^{i}$

$$
0 \subset N_{C / Y}^{0} \subset N_{C / Y}^{1} \subset N_{C / Y}^{2}=N_{C / Y}
$$

one finds subbundles

$$
N_{C / Y}^{i+1} / N_{C / Y}^{i} \subset N_{C / \bar{A}_{g}}^{i+1} / N_{C / \bar{A}_{g}}^{i} .
$$

We arrive at the following definition: 
Definition 1.4. $\varphi: C \rightarrow Y$ satisfies relative Hirzebruch-Höfer proportionality (HHP) if the slope inequalities

$$
\mu\left(N_{C / Y}^{i+1} / N_{C / Y}^{i}\right) \leq \mu\left(N_{C / \bar{A}_{g}}^{i+1} / N_{C / \bar{A}_{g}}^{i}\right), \quad i=0,1,2
$$

are equalities. One has

$$
\begin{aligned}
\mu\left(N_{C / \bar{A}_{g}}^{2} / N_{C / \bar{A}_{g}}^{1}\right) & =\mu\left(S^{2}\left(\mathcal{U}^{\vee}\right)\right)=0, \\
\mu\left(N_{C / \bar{A}_{g}}^{1} / N_{C / \bar{A}_{g}}^{0}\right) & =\mu\left(\mathcal{L}^{-1} \otimes \mathcal{T} \otimes \mathcal{U}^{\vee}\right)=\frac{1}{2} \operatorname{deg} T_{C}\left(-\log S_{C}\right), \\
\mu\left(N_{C / \bar{A}_{g}}^{0}\right) & =\mu\left(T_{C}\left(-\log S_{C}\right)^{\perp}\right)=\operatorname{deg} T_{C}\left(-\log S_{C}\right) .
\end{aligned}
$$

Hence, we obtain a set of inequalities

$$
\begin{aligned}
\mu\left(N_{C / Y}^{2} / N_{C / Y}^{1}\right) & \leq 0, \\
\mu\left(N_{C / Y}^{1} / N_{C / Y}^{0}\right) & \leq \frac{1}{2} \operatorname{deg} T_{C}\left(-\log S_{C}\right), \\
\mu\left(N_{C / Y}^{0}\right) & \leq \operatorname{deg} T_{C}\left(-\log S_{C}\right) .
\end{aligned}
$$

Using $\mu=\frac{\text { deg }}{\text { rank }}$ and adding all three inequalities we obtain a single inequality

$$
\operatorname{deg} N_{C / Y} \leq \frac{\operatorname{rank}\left(N_{C / Y}^{1}\right)+\operatorname{rank}\left(N_{C / Y}^{0}\right)}{2} \cdot \operatorname{deg} T_{C}\left(-\log S_{C}\right) .
$$

It satisfies equality if and only if (HHP) holds.

These conditions are called (HHP) since Hirzebruch [8], in part with Höfer [3], has studied embedded curves on ball quotients and Hilbert modular surfaces and studied proportionality inequalities involving intersection numbers that attain equality if and only if the curve is the compactification of a Shimura curve. Hirzebruch's inequalities together with our proof of them can also be found in [19, Thm. 0.1].

\section{Proposition 1.5.}

(i) If $\varphi: C \rightarrow Y$ satisfies (HHP), then $\varphi^{*} T_{Y}\left(-\log S_{Y}\right)$ is a direct summand of an orthogonal decomposition of $\varphi^{*} T_{\bar{A}_{g}}(-\log S)$ with respect to the Hodge metric. (ii) If $Y^{0} \subset A_{g}$ is a special subvariety, then $\varphi^{*} T_{Y}\left(-\log S_{Y}\right)$ is a direct summand of an orthogonal decomposition of $\varphi^{*} T_{\bar{A}_{g}}(-\log S)$ with respect to the Hodge metric and $\varphi: C \rightarrow Y$ satisfies (HHP). 
Proof. (i) Assuming (HHP), the slope of the sub bundle

$$
N_{C / Y}^{i+1} / N_{C / Y}^{i} \subset N_{C / \bar{A}_{g}}^{i+1} / N_{C / \bar{A}_{g}}^{i}
$$

is equal to the slope of $N_{C / \bar{A}_{g}}^{i+1} / N_{C / \bar{A}_{g}}^{i}$. Since $N_{C / \bar{A}_{g}}^{i+1} / N_{C / \bar{A}_{g}}^{i}$ is polystable, $N_{C / Y}^{i+1} / N_{C / Y}^{i}$ is a direct summand of an orthogonal decomposition of $N_{C / \bar{A}_{g}}^{i+1} / N_{C / \bar{A}_{g}}^{i}$ w.r.t the Hermitian-Yang-Mills metric, which is the induced Hodge metric on $N_{C / \bar{A}_{g}}^{i+1} / N_{C / \bar{A}_{g}}^{i}$.

Claim: The sub bundle $N_{C / Y}^{i} \subset N_{C / \bar{A}_{g}}^{i}, \quad 0 \leq i \leq 2$ is a direct summand and orthogonal.

Proof of the claim. For $i=0$. Since

$$
N_{C / Y}^{0} / N_{C / Y}^{-1}=N_{C / Y}^{0}, \quad N_{C / \bar{A}_{g}}^{0} / N_{C / \bar{A}_{g}}^{-1}=N_{C / \bar{A}_{g}}^{0},
$$

we have shown above

$$
N_{C / Y}^{0} \subset N_{C / \bar{A}_{g}}^{0}
$$

is a direct summand and of an orthogonal decomposition of $N_{C / \bar{A}_{g}}^{0}$ w.r.t.the Hodge metric. Let $p: N_{C / \bar{A}_{g}}^{0} \rightarrow N_{C / Y}^{0}$ denote the projection.

For $i=1$, we consider the following commutative diagramm

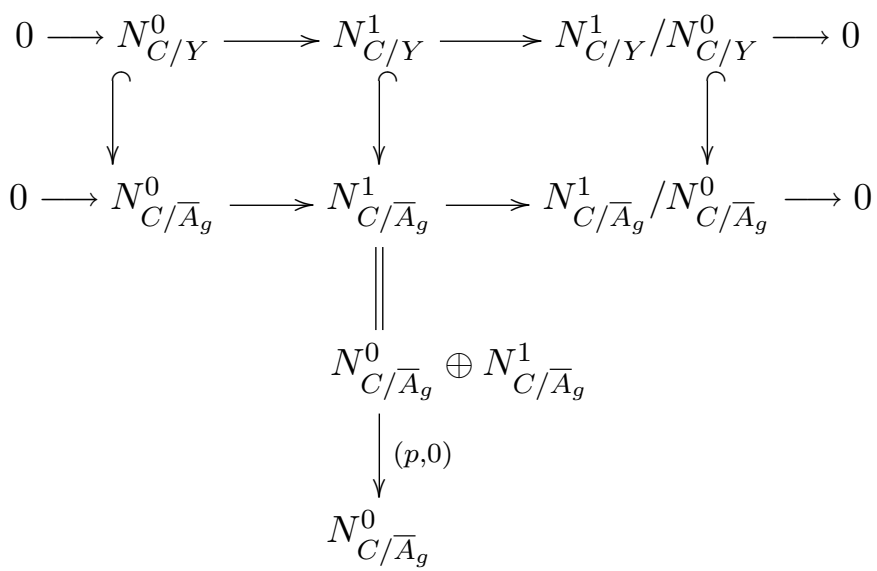

Since the composition map

$$
N_{C / Y}^{0} \rightarrow N_{C / Y}^{1} \rightarrow N_{C / \bar{A}_{g}}^{1} \rightarrow N_{C / \bar{A}_{g}}^{0} \stackrel{p}{\rightarrow} N_{C / Y}^{0}
$$

is the identity, the short exact sequence

$$
0 \rightarrow N_{C / Y}^{0} \rightarrow N_{C / Y}^{1} \rightarrow N_{C / Y}^{1} / N_{C / Y}^{0} \rightarrow 0
$$


splits, and

$$
N_{C / Y}^{1}=N_{C / Y}^{0} \oplus N_{C / Y}^{1} / N_{C / Y}^{0} \subset N_{C / \bar{A}_{g}}^{0} \oplus N_{C / \bar{A}_{g}}^{1} / N_{C / \bar{A}_{g}}^{0}=N_{C / \bar{A}_{g}}^{1} .
$$

Since $N_{C / Y}^{0} \subset N_{C / \bar{A}_{g}}^{0}$ and $N_{C / Y}^{1} / N_{C / Y}^{0} \subset N_{C / \bar{A}_{g}}^{1} / N_{C / \bar{A}_{g}}^{0}$ are direct summands and orthogonal, $N_{C / Y}^{1} \subset N_{C / \bar{A}_{g}}^{1}$ is a direct summand and orthogonal.

Finally, replacing $N^{0}$ by $N^{1} / N^{0}, N^{1}$ by $N^{2}$ and $N^{1} / N^{0}$ by $N^{2} / N^{1}$ in the above diagramm, we obtain $N_{C / Y} \subset N_{C / \bar{A}_{g}}$ is a direct summand and orthogonal. The claim is thus proven.

We are now in the position to finish i). Since by diagram (1.3)

$\varphi^{*} T_{Y}\left(-\log S_{Y}\right)=T_{C}\left(-\log S_{C}\right) \oplus N_{C / Y} \subset T_{C}\left(-\log S_{C}\right) \oplus N_{C / \bar{A}_{g}}=(i \circ \varphi)^{*} T_{\bar{A}_{g}}(-\log S)$, and by the above claim $N_{C / Y} \subset N_{C / \bar{A}_{g}}$ is a direct summand and orthogonal,

$$
\varphi^{*} T_{Y}\left(-\log S_{Y}\right) \subset(i \circ \varphi)^{*} T_{\bar{A}_{g}}(-\log S)
$$

The proof of i) is thus complete.

(ii) Let $i: Y^{0} \hookrightarrow A_{g}$ be a special subvariety. Then $Y^{0}$ is a locally symmetric subvariety of the locally symmetric varity $A_{g}$ and the vector subbundle $d i: T_{Y^{0}} \hookrightarrow$ $i^{*} T_{A_{g}}$ is a locally homogenous subbundle of the locally homogenous bundle $i^{*} T_{A_{g}}$ in the sense of Mumford [20, Sect. 3]. As a locally homogenous bundle can be decomposed as direct sum of irreducible locally homogenous subbundles and this decomposition is orthogonal w.r.t. the invariant metric, $d i: T_{Y^{0}} \hookrightarrow i^{*} T_{A_{g}}$ is a direct summand and orthogonal. Note that the Deligne extension of the sheaf of differential 1-forms is the sheaf of differential 1-forms with logarithmic poles at infinity. By the uniqueness of Deligne's extension we get that $d i: T_{Y}\left(-\log S_{Y}\right) \hookrightarrow$ $i^{*} T_{\bar{A}_{g}}(-\log S)$ is a direct summand and orthogonal. Thus,

$$
d i: \varphi^{*} T_{Y}\left(-\log S_{Y}\right) \subset(i \circ \varphi)^{*} T_{\bar{A}_{g}}(-\log S)
$$

is a direct summand and orthogonal. (The argument here was pointed out by the referee.)

Since $\varphi: C^{0} \rightarrow A_{g}$ is a morphism of Shimura varieties, one has the decomposition

$$
(i \circ \varphi)^{*} T_{\bar{A}_{g}}(-\log S) \simeq S^{2}\left(E^{0,1}\right)=\mathcal{L}^{-2} \otimes S^{2}(\mathcal{T}) \oplus \mathcal{L}^{-1} \otimes \mathcal{T} \otimes \mathcal{U}^{\vee} \oplus S^{2}\left(\mathcal{U}^{\vee}\right)
$$

of polystable subbundles which can be decomposed further as the direct sum of irreducible stable subbundles.

$$
(i \circ \varphi)^{*} T_{\bar{A}_{g}}(-\log S)=K_{1} \oplus \cdots \oplus K_{l} .
$$


By a theorem of Atiyah [2] the category of vector bundles over any compact complex manifold is Krull-Schmidt, i.e., in our case if there is a second decomposition

$$
(i \circ \varphi)^{*} T_{\bar{A}_{g}}(-\log S)=K_{1}^{\prime} \oplus \cdots \oplus K_{l^{\prime}}^{\prime}
$$

of irreducible subbundles, then up to a permutation one has

$$
K_{i} \simeq K_{i^{\prime}}^{\prime}
$$

This shows that $(i \circ \varphi)^{*} T_{Y}\left(-\log S_{Y}\right)$ is the direct sum of some direct factors of $\mathcal{L}^{-2} \otimes S^{2}(\mathcal{T}), \mathcal{L}^{-1} \otimes \mathcal{T} \otimes \mathcal{U}^{\vee}$ and $S^{2}\left(\mathcal{U}^{\vee}\right)$ and therefore the relative proportionality inequality (1.4) is an equality.

In the proof of Proposition 1.5 we see that the inclusion

$$
\varphi^{*} T_{Y}\left(-\log S_{Y}\right) \subset(i \circ \varphi)^{*} T_{\bar{A}_{g}}(-\log S)
$$

is compatible with the decompositions

$$
\begin{aligned}
& \varphi^{*} T_{Y}\left(-\log S_{Y}\right)=T_{C}\left(-\log S_{C}\right) \oplus N_{C / Y}=T_{C}\left(-\log S_{C}\right) \oplus \bigoplus_{i=0}^{1} N_{C / Y}^{i+1} / N_{C / Y}^{i} \\
& \downarrow \quad\|\quad \downarrow \quad\| \quad \\
& (i \circ \varphi)^{*} T_{\bar{A}_{g}}(-\log S)=T_{C}\left(-\log S_{C}\right) \oplus N_{C / \bar{A}_{g}}=T_{C}\left(-\log S_{C}\right) \oplus \bigoplus_{i=0}^{1} N_{C / \bar{A}_{g}}^{i+1} / N_{C / \bar{A}_{g}}^{i}
\end{aligned}
$$

Example 1.6. If $Y$ is a Shimura surface, then $N_{C / Y}$ is a line bundle and there are three cases in which we write (HHP) in terms of more familiar intersection numbers, see [3] and [19, Thm. 0.1]:

(i) $Y$ is a Hilbert modular surface:

$$
N_{C / Y}=N_{C / Y}^{0} \subset \mathcal{L}^{-2} \otimes S^{2}(\mathcal{T}) / \mathcal{L}^{-2}, \quad(H H P): \quad \omega_{Y}(S) \cdot C+2 C^{2}=0 .
$$

(ii) $Y$ is a Picard modular surface:

$$
N_{C / Y} \cong N_{C / Y}^{1} / N_{C / Y}^{0} \subset \mathcal{L}^{-1} \otimes \mathcal{T} \otimes \mathcal{U}^{\vee}, \quad(H H P): \quad \omega_{Y}(S) \cdot C+3 C^{2}=0 .
$$

(iii) $Y$ is product of two Shimura curves:

$$
N_{C / Y} \cong N_{C / Y}^{2} / N_{C / Y}^{1} \subset S^{2}\left(\mathcal{U}^{\vee}\right), \quad(H H P): \quad C^{2}=0,
$$

and $C$ lies in the fibres of one of the projections.

Question 1.7. Does the (HHP) for a single compactified Shimura curve $C$ together with $\varphi: C \rightarrow Y \subset \bar{A}_{g}$ as above imply that $Y^{0} \subset A_{g}$ is a special subvariety, if we assume that the algebraic monodromy group $H\left(Y^{0}\right)$ (see introduction) is $\mathbb{Q}$-simple? 
This question seems to be very optimistic and at the same time difficult to answer. However, we are not aware of any counterexamples if $H\left(Y^{0}\right)$ is $\mathbb{Q}$-simple.

It is our goal in the rest of the paper to show that the existence of "many" special curves, e.g. a dense subset of such satisfying (HHP) forces $Y^{0}$ to be a special subvariety.

Remark 1.8. Consider the same situation $\varphi: C \rightarrow Y \subset \bar{A}_{g}$, where $Y^{0} \subset A_{g}$ is a special subvariety and $C$ an arbitrary curve, not necessarily Shimura. Then one obtains an inequality opposite to (1.3), see for example [19, Thm. 0.3 and Thm. $2.3]$.

\section{Thickening of the Higgs field}

We use the same notation as in the previous section. In particular $C$ is a compactified Shimura curve together with a non-constant morphism $\varphi: C \rightarrow Y \subset \bar{A}_{g}$ factoring over a smooth projective subvariety $Y$ such that $S=\partial \bar{A}_{g}$ intersects $Y$ and the image of $C$ transversely. In the previous section we showed that under these assumptions there is a canonical splitting

$$
\varphi^{*} \Omega_{Y}^{1}\left(\log S_{Y}\right) \cong \Omega_{C}^{1}\left(\log S_{C}\right) \oplus N_{C / Y}^{\vee},
$$

see (1.3). Also we denote by $E=E^{1,0} \oplus E^{0,1}$ the (logarithmic) Higgs bundle on $\bar{A}_{g}$ associated to the local system $\mathbb{V}_{\mathbb{C}}=R^{1} f_{*} \mathbb{C}$, where $f: X \rightarrow A_{g}$ is the universal family over $A_{g}$. Its restriction to $Y$ or $C$ will be denoted by the same symbol. We also make use of the complex vector bundle $\mathcal{V}:=\mathbb{V} \otimes \mathcal{O}_{A_{g}}$ or its restrictions to $Y^{0}$ and $C^{0}$. The following definition is new in the literature and goes back to our discussions with Viehweg. It enables us to include the normal direction to $C$ in $Y$ into our considerations.

Definition 2.1. We define the thickening of the Higgs field $\theta$ on $C$ in the normal direction $N_{C / Y}$ as the pullback of the Higgs bundle on $Y$ via $\varphi: C \rightarrow Y$ :

$$
\theta_{C / Y}:=\varphi^{*} \theta: E^{1,0} \rightarrow E^{0,1} \otimes \varphi^{*} \Omega_{Y}^{1}\left(\log S_{Y}\right)=E^{0,1} \otimes\left(\Omega_{C}^{1}\left(\log S_{C}\right) \oplus N_{C / Y}^{\vee}\right) .
$$

In the same way we define the thickening of the Higgs field in a point $p \in C$ in the normal direction $N_{p / Y}$ as

$\theta_{p / Y}:=\left.\theta_{C / Y}\right|_{p}:\left.\left.\left.E^{1,0}\right|_{p} \rightarrow E^{0,1}\right|_{p} \otimes \varphi^{*} \Omega_{Y}^{1}\left(\log S_{Y}\right)\right|_{p}=\left.E^{0,1}\right|_{p} \otimes\left(\left.\left.\Omega_{C}^{1}\left(\log S_{C}\right)\right|_{p} \oplus N_{C / Y}^{\vee}\right|_{p}\right)$. 
Consider the $k$-fold tensor product $(E, \theta)^{\otimes k}$ of the Higgs bundle $(E, \theta)$ on $Y$. It decomposes as a direct sum

$$
E^{\otimes k}=\bigoplus_{p+q=k} E^{p, q}
$$

where

$$
E^{p, q}=\bigoplus E^{p_{1}, q_{1}} \otimes \cdots \otimes E^{p_{k}, q_{k}}
$$

and where the sum ranges over $p_{i}+q_{i}=1, \sum_{i=1}^{k} p_{i}=p, \sum_{i=1}^{k} q_{i}=q$. The Higgs field, again denoted by $\theta$, decomposes as

$$
\theta: E^{p, q} \rightarrow E^{p-1, q+1} \otimes \Omega_{Y}^{1}\left(\log S_{Y}\right)
$$

where

$E^{p_{1}, q_{1}} \otimes \cdots \otimes E^{p_{k}, q_{k}} \stackrel{\theta}{\longrightarrow} \bigoplus_{i=1}^{k} E^{p_{1}, q_{1}} \otimes \cdots \otimes E^{p_{i}-1, q_{i}+1} \otimes \cdots \otimes E^{p_{k}, q_{k}} \otimes \Omega_{Y}^{1}\left(\log S_{Y}\right)$

satisfies the Leibniz rule

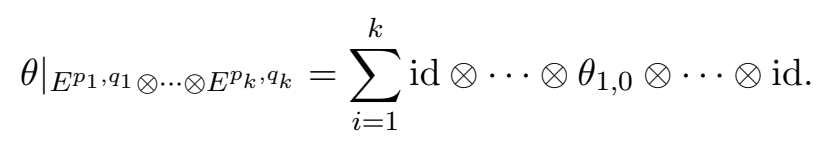

In the same way as in the definition above we define the thickening $\left(E, \theta_{C / Y}\right)^{\otimes k}$ and $\left(E, \theta_{p / Y}\right)^{\otimes k}$.

Assume for a moment that $Y^{0}$ is a locally symmetric quotient of a bounded symmetric domain. Then it is well-known that $\Omega_{Y^{0}}^{1}$, and all Hodge bundles $E_{Y^{0}}^{p, q}$ are locally homogeneous vector bundles in the sense of Mumford [20, Sect. 3]. Furthermore, the Higgs map $\theta^{p, q}:\left.E_{Y^{0}}^{p, q} \rightarrow E^{p-1, q+1}\right|_{Y^{0}} \otimes \Omega_{Y^{0}}^{1}$ is an equivariant morphism between locally homogeneous vector bundles. We decompose $E_{Y^{0}}^{p, q}$ as the direct sum of irreducible locally homogeneous subbundles

$$
E_{Y^{0}}^{p, q}=\bigoplus_{i} E_{Y^{0}, i}^{p, q}
$$

Then we take Mumford's canonical extensions $E_{Y, i}^{p, q}$ [20, Sect. 3], which agrees with Deligne's extension by [15, Lemma 2.4 ] of those irreducible locally homogeneous subbundles, and we use the same symbols

$$
E_{Y}^{p, q}=\bigoplus_{i} E_{Y, i}^{p, q}
$$


for the extended decomposition by uniqueness of good extensions. Complex conjugation

$$
\mathbb{V}_{\mathbb{C}} \stackrel{-}{\rightarrow} \mathbb{V}_{\mathbb{C}}
$$

induces also a complex conjugation on the Deligne extensions of $\left.\mathcal{V}\right|_{C^{0}}=\mathbb{V} \otimes \mathcal{O}_{C^{0}}$ and sends $E_{Y}^{p, q}$ to $E_{Y}^{q, p}\left(\simeq E_{Y}^{p, q \vee}\right)$, hence $E_{Y, i}^{p, q}$ to $E_{Y, i}^{q, p}\left(\simeq E_{Y, i}^{p, q \vee}\right)$.

Given a base point $y \in Y$ ( $y$ could lie on the boundary $S_{Y}$ ) we consider

$$
\theta_{y \in Y}^{p, p}: E_{y}^{p, p} \rightarrow E_{y}^{p-1, p+1} \otimes \Omega_{Y}^{1}\left(\log S_{Y}\right)_{y},
$$

where $E_{y}^{p, p}$ carries the induced real structure from $\mathcal{V}^{\otimes k}$. Its real structure is induced from $\mathbb{V}_{\mathbb{R}}=\mathbb{V}_{\mathbb{Q}} \otimes \mathbb{R}$.

Definition 2.2. Let $y \in Y$ be a base point:

(a) $W_{y \in Y}:=\left\{t \in E_{y}^{p, p} \mid \theta_{y \in Y}(t)=0\right\}$.

(b) A tensor $t \in E_{y}^{p, p} \cap \mathcal{V}_{\mathbb{R}, y}^{\otimes k}$ is called a real Hodge tensor at the base point $y \in Y$.

(c) $W_{y \in Y, \mathbb{R}}:=\left\{t \in E_{y}^{p, p} \cap \mathcal{V}_{\mathbb{R}, y}^{\otimes k} \mid \theta_{y \in Y}(t)=0\right\}$.

Remark 2.3. It is clear that $W_{y \in Y, \mathbb{R}} \otimes \mathbb{C} \subset W_{y \in Y}$, but in general they are not equal.

Proposition 2.4 (Parallel Transport). There exists a unitary subsystem $\mathbb{W} \subset$ $\mathbb{V}^{\otimes k}$ of pure Hodge type $(p, p)$, which naturally extends to $Y$, carries an induced real structure from $\mathbb{V}^{\otimes k}$ and such that $\mathbb{W}_{\mathbb{R}, y}=W_{y \in Y, \mathbb{R}}$ for all $y \in Y$.

Proof. From the above discussion we know that $E_{Y}^{p, p}$ decomposes as direct sum of good extensions of irreducible locally homogeneous subbundles

$$
E_{Y}^{p, p}=\bigoplus_{i} E_{Y, i}^{p, p}
$$

Since $\theta^{p, p}: E^{p, p} \rightarrow E^{p-1, p+1} \otimes \Omega_{Y}^{1}\left(\log S_{Y}\right)$ is a morphism between good extensions of locally homogeneous vector bundles, $\operatorname{ker}\left(\theta^{p, p}\right)$ is again a direct sum of $\operatorname{good}$ extensions of locally homogeneous subvector bundles, which are Higgs subbundles (with trivial Higgs field) of $(E, \theta)^{\otimes k}$. By Simpson's polystability all of them have non-positive slopes. We decompose (as holomorphic vector bundles)

$$
\operatorname{ker}\left(\theta^{p, p}\right)=\operatorname{ker}\left(\theta^{p, p}\right)_{0} \oplus \operatorname{ker}\left(\theta^{p, p}\right)_{<0},
$$

where $\operatorname{ker}\left(\theta^{p, p}\right)_{0}$ is the direct sum of good extensions of locally homogenous subvector bundles of slope zero and $\operatorname{ker}\left(\theta^{p, p}\right)_{<0}$ is the direct sum of good extensions 
of locally homogeneous subvector bundles of negative slopes. By Simpson's correspondence $\operatorname{ker}\left(\theta^{p, p}\right)_{0}$ underlies a unitary local subsystem $\mathbb{W} \subset \mathbb{V} \otimes k$ of type $(p, p) . \operatorname{ker}\left(\theta^{p, p}\right)_{0}$ is invariant under complex conjugation induced by the real structure on $\mathbb{V}^{\otimes k}$. This can be seen as follows: the complex conjugate $\overline{\operatorname{ker}\left(\theta^{p, p}\right)_{0}}$ corresponds to the complex conjugate $\overline{\mathbb{W}}$ of $\mathbb{W}$, which is again a unitary sublocal system of type $(p, p)$. Hence it vanishes under the Higgs field $\theta^{p, p}$, i.e., $\overline{\operatorname{ker}\left(\theta^{p, p}\right)_{0}} \subset \operatorname{ker}\left(\theta^{p, p}\right)$. Note that $\overline{\operatorname{ker}\left(\theta^{p, p}\right)_{0}}$ is again the direct sum of some good extensions of locally homogeneuos subvector bundles of slope zero, hence we obtain $\overline{\operatorname{ker}\left(\theta^{p, p}\right)_{0}} \subset \operatorname{ker}\left(\theta^{p, p}\right)_{0}$. Clearly for all real vector $t \in \operatorname{ker}\left(\theta^{p, p}\right)_{0, y}$ we have $\theta_{y}^{p, p}(t)=0$, so $t \in W_{y \in Y, \mathbb{R}}$. Conversely, let $t \in W_{y \in Y, \mathbb{R}}$. Then $t$ is a real vector in $\operatorname{ker}\left(\theta^{p, p}\right)_{y}$. There are no vectors in $\operatorname{ker}\left(\theta^{p, p}\right)_{<0, y}$ fixed by complex conjugation, because complex conjugation takes negative slopes to positive slopes. Therefore $t$ is a real vector in $\operatorname{ker}\left(\theta^{p, p}\right)_{0, y}$. Thus we have shown

$$
\mathbb{W}_{\mathbb{R}, y}=W_{y \in Y, \mathbb{R}}
$$

Since $\mathbb{V}^{\otimes k}$ has unipotent local monodromies around $S_{Y}$ and $\mathbb{W}$ is unitary, the local monodromies of $\mathbb{W}$ are trivial around $S_{Y}$. Hence $\mathbb{W}$ extends across $S_{Y}$.

Remark 2.5. For a rational Hodge tensor $t \in W_{y \in Y}, t$ is contained in a unitary local subsystem with a $\mathbb{Z}$-structure. Hence the orbit

$$
\left\{\rho(\gamma)(t) \mid \gamma \in \pi_{1}\left(Y^{0}, y\right)\right\}
$$

is finite.

For a Shimura curve $C^{0}$ mapping to $A_{g}$ via $\varphi$ as above we can describe the above decomposition more precisely. For the Higgs bundle $E$ one has from section 1

$$
E^{1,0}=\mathcal{L} \otimes \mathcal{T} \oplus \mathcal{U}, \quad E^{0,1}=\mathcal{L}^{-1} \otimes \mathcal{T} \oplus \mathcal{U}^{\vee},
$$

where $\mathcal{L} \otimes \mathcal{T}$ and $\mathcal{L}^{-1} \otimes \mathcal{T}$ are polystable of slopes $\operatorname{deg} \mathcal{L}$ and $-\operatorname{deg} \mathcal{L}$ respectively. Moreover if $\mathcal{U} \neq 0$ then $\mathcal{U}$ and $\mathcal{U}^{\vee}$ are both polystable of slope zero. So one obtains immediately:

Lemma 2.6. The sheaves $E^{p, q}$ are direct sums of polystable sheaves $E_{\iota}^{p, q}$ of slopes $\mu\left(E_{\iota}^{p, q}\right)=\iota \operatorname{deg} \mathcal{L}$ and one has:

(a) If $\mathcal{U}=0$, then $E_{\iota}^{p, q} \neq 0$ if and only if $\iota=p-q$, and $E^{p, q}=E_{\iota}^{p, q}$.

(b) If $\mathcal{U} \neq 0$, then $E_{\iota}^{p, q} \neq 0$ if and only if $\iota \in\{-q, \ldots, p\}$. In this case $E_{\iota}^{p, q}$ is a 
direct sum of sheaves of the form

$$
\bigoplus_{m-l=\iota}(\mathcal{L} \otimes \mathcal{T})^{\otimes m} \otimes\left(\mathcal{L}^{-1} \otimes \mathcal{T}\right)^{\otimes l} \otimes \mathcal{U}^{\otimes(p-m)} \otimes \mathcal{U}^{\vee \otimes(q-l)}
$$

(c) The sheaf $E_{\iota}^{p, p}$ is dual to $E_{-\iota}^{p, p}$.

Consider the decomposition (1.3)

$$
\varphi^{*} T_{Y}\left(-\log S_{Y}\right)=N_{C / Y} \oplus T_{C}\left(-\log S_{C}\right) .
$$

The assumption that $\varphi: C \rightarrow Y$ satisfies (HHP) implies the decomposition in the proof of Proposition 1.2

$$
N_{C / Y}=N_{C / Y}^{0} \oplus N_{C / Y}^{1} / N_{C / Y}^{0} \oplus N_{C / Y}^{2} / N_{C / Y}^{1}
$$

such that

$$
\begin{aligned}
& N_{C / Y}^{0} \subset N_{C / \bar{A}_{g}}^{0}=\mathcal{L}^{-2} \otimes S^{2}(\mathcal{T}) / \mathcal{L}^{-2} \subset \mathcal{H o m}\left(\mathcal{L} \otimes \mathcal{T}, \mathcal{L}^{-1} \otimes \mathcal{T}\right) / \mathcal{L}^{-2}, \\
& N_{C / Y}^{1} / N_{C / Y}^{0} \subset N_{C / \bar{A}_{g}}^{1} / N_{C / \bar{A}_{g}}^{0}=\mathcal{L}^{-1} \otimes \mathcal{T} \otimes \mathcal{U}^{\vee}=\mathcal{H o m}\left(\mathcal{L} \otimes \mathcal{T}, \mathcal{U}^{\vee}\right)
\end{aligned}
$$

and

$$
N_{C / Y}^{2} / N_{C / Y}^{1} \subset N_{C / \bar{A}_{g}}^{2} / N_{C / \bar{A}_{g}}^{1}=S^{2}\left(\mathcal{U}^{\vee}\right) \subset \mathcal{H o m}\left(\mathcal{U}, \mathcal{U}^{\vee}\right)
$$

are direct polystable factors of slopes $-2 \operatorname{deg} \mathcal{L}$, respectively $-\operatorname{deg} \mathcal{L}$, respectively 0 . In this way we may decompose the thickening $\theta_{C / Y}$ in the form

$$
\theta_{C / Y}=\theta_{C}+\theta_{N_{C / Y}}=\theta_{C}+\theta_{N_{C / Y}^{0}}+\theta_{N_{C / Y}^{1} / N_{C / Y}^{0}}+\theta_{N_{C / Y}^{2} / N_{C / Y}^{1}} .
$$

Using that decomposition we obtain:

Lemma 2.7. The thickening $\theta_{C / Y}$ on $E_{\iota}^{p, q}$ can be decomposed as a direct sum of morphisms:

$$
\begin{aligned}
& E_{\iota}^{p, q} \stackrel{\theta_{C}+\theta_{N_{C / Y}^{0}}}{\longrightarrow} E_{\iota-2}^{p-1, q+1} \otimes\left(\Omega_{C}^{1}\left(\log S_{C}\right) \oplus N_{C / Y}^{0} \vee,\right. \\
& E_{\iota}^{p, q} \stackrel{\theta_{N_{C / Y}^{1} / N_{C / Y}^{0}}^{\longrightarrow}}{\longrightarrow} E_{\iota-1}^{p-1, q+1} \otimes\left(N_{C / Y}^{1} / N_{C / Y}^{0}\right)^{\vee}
\end{aligned}
$$

and

$$
E_{\iota}^{p, q} \stackrel{\theta_{N_{C / Y}^{2} / N_{C / Y}^{1}}^{\longrightarrow}}{\longrightarrow} E_{\iota}^{p-1, q+1} \otimes\left(N_{C / Y}^{2} / N_{C / Y}^{1}\right)^{\vee} .
$$

between polystable sheaves of the same slopes. 
Proof. Write

$$
E^{1,0}=\mathcal{L} \otimes \mathcal{T} \oplus \mathcal{U}, \quad E^{0,1}=\mathcal{L}^{-1} \otimes \mathcal{T} \oplus \mathcal{U}^{\vee},
$$

then $\theta_{C / Y}: E^{1,0} \rightarrow E^{0,1} \otimes \varphi^{*} \Omega_{Y}^{1}\left(\log S_{Y}\right)$ decomposes into the following terms:

$$
\begin{gathered}
\mathcal{L} \otimes \mathcal{T} \stackrel{\theta_{C}}{\longrightarrow} \mathcal{L}^{-1} \otimes \mathcal{T} \otimes \Omega_{C}^{1}\left(\log S_{C}\right), \\
\mathcal{L} \otimes \mathcal{T} \stackrel{{ }_{N_{C / Y}^{0}}}{\longrightarrow} \mathcal{L}^{-1} \otimes \mathcal{T} \otimes N_{C / Y}^{0 \vee}, \\
\mathcal{L} \otimes \mathcal{T} \stackrel{{ }_{N_{C / Y}^{1} / N_{C / Y}^{0}}}{\longrightarrow} \mathcal{U}^{\vee} \otimes\left(N_{C / Y}^{1} / N_{C / Y}^{0}\right)^{\vee}
\end{gathered}
$$

and

$$
\mathcal{U} \stackrel{\theta_{N_{C / Y}^{2}} / N_{C / Y}^{1}}{\longrightarrow} \mathcal{U}^{\vee} \otimes\left(N_{C / Y}^{2} / N_{C / Y}^{1}\right)^{\vee}
$$

This proves the lemma for the case $k=1$. In general, one reduces the cases $k \geq 2$ to the case $k=1$ using the fact that the thickening $\theta_{C / Y}^{\otimes k}$ is defined by the Leibniz rule.

\section{Parallel transport of Real Hodge tensors on Connected Cycles OF SPECIAL CURVES}

In this section let $Y \subset \bar{A}_{g}$ be a smooth projective subvariety, which meets $S=$ $\partial \bar{A}_{g}$ transversely. Assume $Y$ contains a connected cycle $\sum_{i} C_{i}$ of finitely many compactified embedded special curves, such that each component $C_{i}$ meets $S_{Y}=$ $S \cap Y$ transversely and satisfies (HHP). Using base points $y_{i} \in C_{i}^{0}$ and notations from the previous section we introduce the following subspaces:

$$
\begin{aligned}
W_{y_{i} \in Y} & :=\left\{t \in E_{y_{i}}^{p, p} \mid \theta_{y_{i} \in Y}(t)=0\right\} \\
\cap & \\
W_{y_{i} \in C_{i}} & :=\left\{t \in E_{y_{i}}^{p, p} \mid \theta_{y_{i} \in C_{i}}(t)=0\right\}
\end{aligned}
$$

and the real spaces

$$
\begin{aligned}
W_{y_{i} \in Y, \mathbb{R}} & :=\left\{\left.t \in E_{y_{i}}^{p, p} \cap \mathcal{V}_{\mathbb{R}}^{\otimes k}\right|_{y_{i}} \mid \theta_{y_{i} \in Y}(t)=0\right\}, \\
\cap & \\
W_{y_{i} \in C_{i}, \mathbb{R}} & :=\left\{\left.t \in E_{y_{i}}^{p, p} \cap \mathcal{V}_{\mathbb{R}}^{\otimes k}\right|_{y_{i}} \mid \theta_{y_{i} \in C_{i}}(t)=0\right\} .
\end{aligned}
$$

Fixing a base point $y_{1} \in C_{1}^{0}$ we now need to study the parallel transport of real vectors in $W_{y_{1} \in Y, \mathbb{R}}$ along paths in the connected subspace $\bigcup_{i} C_{i}$. 
Proposition 3.1. (a) The real subspace $W_{y_{1} \in Y, \mathbb{R}} \subset \mathbb{V}_{\mathbb{R}}^{\otimes k}$ is invariant under the monodromy action $\rho^{\otimes k}\left(\pi_{1}\left(\bigcup_{i} C_{i}^{0}, y_{1}\right)\right)$.

(b) Assume that $E_{C_{i}}^{p, p}$ is polystable of slope zero for all $C_{i}$. Then $W_{y_{1} \in Y}$ is invariant under the monodromy action $\rho^{\otimes k}\left(\pi_{1}\left(\bigcup_{i} C_{i}^{0}, y_{1}\right)\right)$.

Proof. (a) We have the decomposition

$$
\theta_{y_{1} / Y}=\theta_{y_{1} / C_{1}} \oplus \theta_{N_{C_{1} / Y}, y_{1}}
$$

Hence,

$$
W_{y_{1} \in Y, \mathbb{R}}=\left\{t \in W_{y_{1} \in C_{1}, \mathbb{R}} \mid \theta_{N_{C_{1} / Y}, y_{1}}=0\right\} .
$$

By Proposition 2.4 there exists a unitary subsystem $\mathbb{W}_{C_{1}} \subset \mathbb{V}_{\mathbb{R}}^{\otimes k}$ of Hodge type $(p, p)$ such that $W_{y_{1} \in C_{1}, \mathbb{R}}=\mathbb{W}_{C_{1}, y_{1}}$.

Let $\mathcal{W}_{C_{1}} \subset E_{C_{1}}^{p, p}$ denote the polystable subbundle of slope zero corresponding to $\mathbb{W}_{C_{1}}$. Then by Lemma 2.7

$$
\theta_{N_{C_{1} / Y}}: \mathcal{W}_{C_{1}} \rightarrow \theta_{N_{C_{1} / Y}}\left(\mathcal{W}_{C_{1}}\right)
$$

is a morphism between polystable bundles of slope zero. Hence the kernel

$$
\operatorname{ker}\left(\theta_{N_{C_{1} / Y}}: \mathcal{W}_{C_{1}} \rightarrow \theta_{N_{C_{1} / Y}}\left(\mathcal{W}_{C_{1}}\right)\right)=: \mathcal{W}_{C_{1}}^{\prime}
$$

is a polystable subbundle of $\mathcal{W}_{C_{1}}$ of slope zero. Therefore it underlies a unitary subsystem $\mathbb{W}_{C_{1}}^{\prime} \subset \mathbb{W}_{C_{1}} \otimes \mathbb{C}$. From the construction of $\mathbb{W}_{C_{1}}^{\prime}$ we see that

$$
W_{y_{1} \in Y, \mathbb{R}} \subset \mathbb{W}_{C_{1}, y_{1}}^{\prime} .
$$

We start with a real vector $t_{1} \in W_{y_{1} \in Y, \mathbb{R}}$ and denote by $t_{2}$ the parallel transport of $t_{1}$ as a vector in the fibre of the local system $\mathbb{V}_{\mathbb{R}, y_{1}}^{\otimes k}$ along some path in $C_{1}^{0}$ from $y_{1}$ to $y_{2} \in C_{1}^{0} \cap C_{2}^{0}$. Since $t_{1}$ is contained in the fibre of the subsystem $\mathbb{W}_{C_{1}}^{\prime} \subset \mathbb{V}_{\mathbb{C}, C_{1}}^{\otimes k}$ at $y_{1}, t_{2}$ is a real vector (because of the real structure on $\mathbb{V}^{\otimes k}$ ) and contained in the fibre of $\mathbb{W}_{C_{1}}^{\prime}$ at $y_{2}$. By the construction of $\mathbb{W}_{C_{1}}^{\prime}$, we see that $\theta_{y_{2} / C_{1}}\left(t_{2}\right)=0$ and $\theta_{N_{C_{1} / Y}, y_{2}}\left(t_{2}\right)=0$, i.e., $t_{2} \in W_{y_{2} \in Y, \mathbb{R}}$.

Regarding $y_{2} \in C_{2}^{0}$ we repeat the above argument and continue the parallel transport of $t_{2}$ along some path in $C_{2}^{0}$ from $y_{2}$ to $y_{3} \in C_{2} \cap C_{3}$ etc.. This shows that $W_{y_{1} \in Y, \mathbb{R}} \subset \mathbb{V}_{\mathbb{R}, y_{1}}^{\otimes k}$ is invariant under the monodromy action $\rho^{\otimes k}\left(\pi_{1}\left(\bigcup_{i} C_{i}^{0}, y_{1}\right)\right)$.

(b) Since $E_{C_{1}}^{p, p}$ is polystable of slope zero, $\operatorname{ker}\left(\theta_{C_{1}}^{p, p}\right)$ is a Higgs subbundle of slope zero (with trivial Higgs field) and it corresponds to an (extended) unitary local system $\mathbb{W}_{C_{1}} \subset \mathbb{V}_{C_{1}}^{\otimes k}$ with an induced real structure, and such that $\mathbb{W}_{C_{1}, y}=$ 
$W_{y \in C_{1}}$ for all $y \in C_{1}$. Since $W_{y_{1} \in Y}=\left\{t \in W_{y_{1} \in C_{1}} \mid \theta_{N_{C_{1} / Y}, y_{1}}(t)=0\right\}$, by the same argument as in (a) we find a unitary subsystem $\mathbb{W}_{C_{1}}^{\prime} \subset \mathbb{W}_{C_{1}}$ such that $\mathbb{W}_{C_{1}, y}^{\prime}=W_{y \in Y}$ for all $y \in C_{1}$. The rest of the proof is exactly the same as in (a).

Definition 3.2. We say that $Y^{0}$ can be covered by a smoothing of a cycle $\sum_{i} a_{i} C_{i}$ of special curves $C_{i}$ satisfying (HHP), if there is a suitable linear combination $\sum_{i} a_{i} C_{i}$ of compactified special curves $C_{i} \subset Y$ satisfying (HHP) which can be deformed into a generically smooth family of curves $\cup_{z \in Z} C_{z}$ filling out $Y$, i.e., such that $\sum_{i} a_{i} C_{i}$ is a degenerate fibre of a generically smooth family of curves $\cup_{z \in Z} C_{z}$ over some parameter scheme $Z$.

Proposition 3.3. Assume that the algebraic monodromy group $H\left(Y^{0}\right)$ of $Y^{0}$ (defined in the introduction) is $\mathbb{Q}$-simple and that $Y^{0}$ can be covered by a smoothing of a cycle $\sum_{i} a_{i} C_{i}$ of special curves $C_{i}$ satisfying (HHP). We fix a base point $y_{0} \in C_{1}^{0}$.

(a) Then $W_{y_{1} \in Y, \mathbb{R}} \subset \mathbb{V}_{\mathbb{R}, y_{1}}^{\otimes k}$ is $\rho^{\otimes k}\left(\pi_{1}\left(Y^{0}, y_{0}\right)\right)$-invariant.

(b) Under the assumption in Prop. 3.1 (b), $W_{y_{0} \in Y} \subset \mathbb{V}_{\mathbb{C}, y_{1}}^{\otimes k}$ is $\rho^{\otimes k}\left(\pi_{1}\left(Y^{0}, y_{0}\right)\right)$ invariant.

The following Lemma is Proposition 2.2.2 in [26]. It will be used below.

Lemma 3.4. Let $X$ be a smooth complex quasi-projective variety, $k$ a field of characteristic 0, $G$ an almost simple $k$-algebraic group and

$$
\rho: \pi_{1}(X, *) \rightarrow G(k)
$$

be a Zariski dense representation. Then the following holds:

(1) If $\pi: X^{\prime} \rightarrow X$ is a surjective and generically finite morphism, and $X^{\prime}$ is smooth, then $\pi^{*}(\rho)$ is again Zariski dense.

(2) If $f: X \rightarrow Y$ is a surjective morphism to a smooth quasi-projective variety $Y$ with connected fibres, and if $f^{-1}(y) \subset X$ is a smooth fibre, then there are two possibilities:

either

(i) the restriction $\left.\rho\right|_{f^{-1}(y)}$ is again Zariski dense, or

(ii) $\left.\rho\right|_{f^{-1}(y)}$ has finite image.

Proof. (Proposition 3.3) (a) Fix a smooth curve $C_{z}$ in the family $\cup_{z \in Z} C_{z}$ and a base point $* \in C_{z}$. Then $C_{z}^{0}$ deformes to $\sum_{i} a_{i} C_{i}^{0}$ and $*$ moves to $y_{0} \in \sum_{i} C_{i}^{0}$ 
along a path $\gamma_{* y_{0}}$. This implies that any loop lying on $C_{z}^{0}$ with base point $*$ is homotopic to some loop lying on $\sum_{i} C_{i}^{0}$ with base point $y_{0}$. By Proposition 3.1 the induced representation

$$
\rho_{C_{z}^{0}}: \pi_{1}\left(C_{z}^{0}, *\right) \rightarrow \pi_{1}\left(Y^{0}, *\right) \stackrel{\rho^{\otimes k}}{\rightarrow} \mathbb{V}_{*}^{\otimes k}
$$

stabilizes the real subspace of $W_{*} \subset E_{\mathbb{R}, *}^{p, p}$ which is the parallel transport of $W_{y_{0} \in Y}$ along the path $\gamma_{* y_{\circ}}^{-1}$.

By assumption, the algebraic monodromy group $H\left(Y^{0}\right)$ is $\mathbb{Q}$-simple. The covering family is given by a correspondence in $Y \times Z$ and can be chosen such that there are finitely many curves through a generic point of $Y$. Therefore, after taking a generically finite base change $Y^{0^{\prime}} \rightarrow Y^{0}$, we may assume that the family gives rise to a surjective map $g: Y^{0} \rightarrow Z^{0}$ with connected fibres, and such that $C_{z}^{0} \subset Y^{0}$ is a smooth fibre of $g$. Note that this modification does not change the algebraic monodromy group $H\left(Y^{0}\right)$ by (1) in Lemma 3.4 .

By (2) in Lemma 3.4 there are two possibilities: either (i): $H\left(C_{z}^{0}\right)=H\left(Y^{0}\right)$, or (ii): $H\left(C_{z}^{0}\right)$ is a finite group. The case (ii) is impossible. Otherwise the restricted representation $\rho_{C_{z}^{0}}: \pi_{1}\left(C_{z}^{0} *\right) \rightarrow S p(2 g, \mathbb{Q})$ would have finite image, which implies that the restricted period map $\varphi: C_{z}^{0} \rightarrow A_{g}$ is constant. A contradiction.

So we obtain $H\left(C_{z}^{0}\right)=H\left(Y^{0}\right)$. Since $\rho_{C_{z}^{0}}^{\otimes k}$ stabilizes $W_{*}$, which is indeed an algebraic condition for the monodromy matrices of $\rho_{C_{z}^{0}}$, the representation $H\left(C_{z}^{0}\right)$ in $\mathbb{V}_{*}^{\otimes k}$ also stabilizes $W_{*}$. This shows that $\rho^{\otimes k}\left(\pi_{1}\left(Y^{0}, *\right)\right)$ stabilizes $W_{*}$. Now by moving the base point $*$ along the path $\gamma_{* y_{0}}$ to $y_{0}$ we obtain that $W_{y_{0} \in Y, \mathbb{R}}$ is $\rho^{\otimes k}\left(\pi_{1}\left(Y^{0}, y_{0}\right)\right)$-invariant. The proof of (b) is the same as the one of (a).

Corollary 3.5. Assume $W_{y_{0} \in Y}$ carries a $\mathbb{Q}$-structure from $\mathbb{V}^{\otimes k}$. Then the subsystem $\mathbb{U}_{Y^{0}}^{p, p}$ has finite monodromy and $W_{y_{0} \in Y}$ extends to a subspace of sections of $\mathbb{V}_{Y^{0}}^{\otimes k}$.

We assume now $Y^{0}$ is contained in a Shimura subvariety $M^{0} \subset A_{g}$ of type $S O(2, n)$ with toroidal compactification $M \subset \bar{A}_{g}$. Then $\mathbb{V}_{M^{0}}^{\otimes 2}$ contains a sub-VHS of Hodge structures $\mathbb{V}^{\prime}$, whose corresponding Higgs bundle has the form

$$
E=E^{2,0} \oplus E^{1,1} \oplus E^{0,2}, \quad \theta^{2,0}: T_{M}\left(-\log S_{M}\right) \otimes E^{2,0} \simeq E^{1,1}, \quad \theta^{1,1}=\theta^{2,0 \vee} .
$$

The Griffiths-Yukawa coupling for $E$ along any subvariety $Z \subset M$ meeting $S_{M}$ transversely is the iterated Kodaira-Spencer derivative

$$
E^{2,0} \longrightarrow E^{0,2} \otimes S^{2} \Omega_{Z}^{1}\left(\log S_{Z}\right)
$$


The following statements and proofs will use this notation.

Theorem 3.6. Let $Y \subset \bar{A}_{g}$ be as above. Assume that $Y^{0}$ is contained in a Shimura subvariety $M^{0} \subset A_{g}$ of type $S O(2, n)$. We assume that $Y$ and $M$ intersect the boundary $S$ of $A_{g}$ transversely, and that $Y$ can be covered by a smoothing of a cycle $\sum_{i} a_{i} C_{i}$ of special curves $C_{i} \subset Y$ satisfying (HHP). Then:

(a) If $W_{y_{0} \in Y}=W_{y_{0} \in Y, \mathbb{R}} \otimes \mathbb{C}$ for some $y_{0} \in C_{1}$ then $Y^{0} \subset M^{0}$ is a special subvariety of orthogonal type.

(b) If the Griffiths-Yukawa couplings along all $C_{i}$ do not vanish then $Y^{0} \subset M^{0}$ is a special subvariety of orthogonal type.

(c) If the Griffiths-Yukawa coupling along $Y$ vanishes then $Y^{0} \subset M^{0}$ is a special subvariety of unitary type, i.e., a ball quotient.

Remark 3.7. It is not hard to show that the assumptions of the theorem are necessary, since by Borcherds' results [4] any Shimura variety of type $S O(2, n)$ contains sections of powers of automorphic line bundles which are unions of orthogonal special subvarieties and components of $S_{M}$.

One can show that $\Omega_{Y}^{1}\left(\log S_{Y}\right)$ is nef on $Y$, and $\omega_{Y}\left(S_{Y}\right)$ is ample with respect to $Y^{0}$. This follows from our transversality assumptions.

In the assertions (a) and (b) of the theorem one may replace the assumption on the smoothing of the cycle $\sum_{i} a_{i} C_{i}$ of special curves $C_{i} \subset Y$ satisfying (HHP) by the following: Assume that there is a connected union $C_{1} \cup \cdots \cup C_{l}$ of special curves satisfying (HHP) and such that the image of

$$
\pi_{1}\left(\bigcup C_{i}^{0}, *\right) \longrightarrow \pi_{1}\left(Y^{0}, *\right)
$$

has finite index for some basepoint $*$.

Proof. $Y^{0}$ is contained in $M^{0}$, which is a Shimura variety for $S O(2, n)$ without compact factors. All Hermitian type subgroups of $S O(2, n)$ except $G=\mathrm{SL}_{2} \times \mathrm{SL}_{2}$ and quaternionic versions (see main theorem in [21]) are $\mathbb{Q}$-simple for rank reasons and either orthogonal or unitary. Hence $H\left(Y^{0}\right)$ will be $\mathbb{Q}$-simple unless $\operatorname{dim}(Y)=2$. In that case it follows that $Y^{0}$ is uniformized by a product $\mathbb{H} \times \mathbb{H}$ of upper half planes. For the rest of the proof we may therefore assume that $H\left(Y^{0}\right)$ is $\mathbb{Q}$-simple and $\operatorname{dim}(Y) \geq 3$.

(a) By Prop. 3.3 (a) the real subspace $W_{y_{0} \in Y, \mathbb{R}}$ is $\rho^{\otimes 2}\left(\pi_{1}\left(Y^{0}, y_{0}\right)\right)$-invariant. Hence $W_{y_{0} \in Y, \mathbb{R}} \otimes \mathbb{C}$ defines a unitary subsystem $\mathbb{U}$ of the local system $\mathbb{V}_{Y, \mathbb{C}}^{\prime}$ underlying the Higgs bundle $E$ (see introduction) and a corresponding decomposition 
of Higgs bundles

$$
\left(E_{Y}^{2,0} \oplus E_{Y}^{1,1^{\prime}} \oplus E_{Y}^{0,2}, \theta_{Y}\right) \oplus\left(E_{Y}^{1,1^{\prime \prime}}, 0\right) .
$$

Note that $\theta_{Y}: E_{Y}^{1,1} \rightarrow E_{Y}^{0,2} \otimes \Omega_{Y}^{1}\left(\log S_{Y}\right)$ is surjective, since the pair $\left(Y, S_{Y}\right)$ is transversely embedded in $\left(\overline{A_{g}}, S\right)$. Therefore we have $\operatorname{rank} E_{Y}^{1,1^{\prime}}=\operatorname{dim} Y$, and

$$
\operatorname{rank} E_{Y}^{1,1^{\prime \prime}}=\operatorname{dim} W_{y_{0} \in Y, \mathbb{R}} \otimes \mathbb{C}=\operatorname{dim} W_{y_{0} \in Y}=\operatorname{dim} M-\operatorname{dim} Y .
$$

This implies that

$$
\theta_{Y}: T_{Y}\left(-\log S_{Y}\right) \otimes E_{Y}^{2,0} \rightarrow E_{Y}^{1,1^{\prime}}
$$

is an isomorphism. Hence the image of $Y^{0}$ in $A_{g}$ is a locally symmetric quotient of the period domain $D$ of orthogonal type associated to the complement $\mathbb{U}^{\perp}$ of $\mathbb{U}$ in $\mathbb{V}^{\prime}$. As a consequence, $Y^{0} \hookrightarrow M^{0}$ is a totally geodesic embedding. Together with the rigidity of $Y^{0} \subset M^{0}$, which follows from $\operatorname{dim}(Y) \geq 2$ [19, Lemma 1.5], we obtain that $Y^{0} \subset M^{0}$ is a special subvariety of orthogonal type by the arguments in loc. cit..

(b) We will give two proofs. First Proof: The non-vanishing of the GriffithsYukawa coupling along $C_{i}$ implies that

$$
\mathbb{V}_{C_{i}}^{\prime}=\mathbb{V}^{\prime \prime} \oplus \mathbb{U}^{1,1}
$$

where $\mathbb{V}^{\prime \prime}$ is a sub-VHS with rank one Hodge bundles

$$
E_{C_{i}}^{2,0} \oplus E_{C_{i}}^{1,1^{\prime}} \oplus E_{C_{i}}^{0,2}:=E_{C_{i}}^{2,0} \oplus \theta\left(E_{C_{i}}^{2,0}\right) \oplus \theta^{2}\left(E_{C_{i}}^{2,0}\right),
$$

and $U_{C_{i}}^{1,1}$ is a sub-VHS of pure Hodge type $(1,1)$. Hence $E_{C_{i}}^{1,1}$ is polystable of slope zero. Fix a base point $y_{0} \in C_{1}^{0}$. Then by Proposition 3.3 (b) the subspace $W_{y_{0} \in Y} \subset \mathbb{V}_{\mathbb{C}, y_{0}}^{\prime}$ is $\rho^{\otimes 2}\left(\pi_{1}\left(Y^{0}, y_{0}\right)\right)$-invariant. Hence the Higgs bundle $E$ associated to $\mathbb{V}_{Y^{0}}^{\prime}$ decomposes as

$$
\left(E_{Y}^{2,0} \oplus E_{Y}^{1,1^{\prime}} \oplus E_{Y}^{0,2}, \theta_{Y}\right) \oplus\left(E_{Y}^{1,1^{\prime \prime}}, 0\right),
$$

where the Higgs subbundle $\left(E_{Y}^{1,1^{\prime \prime}}, 0\right)$ corresponds to the unitary subsystem of rank equal to $\operatorname{dim} M-\operatorname{dim} Y$ defined above. This implies that

$$
\theta_{Y}: T_{Y}\left(-\log S_{Y}\right) \otimes E_{Y}^{2,0} \rightarrow E_{Y}^{1,1^{\prime}}
$$

is an isomorphism. As in (a), $Y^{0} \subset M^{0}$ is a totally geodesic embedding and the rigidity of $Y^{0} \subset M^{0}$ implies that $Y^{0} \subset M^{0}$ is a special subvariety of orthogonal type.

Second proof for (b): Let $\left(F_{Y}, \theta_{Y}\right) \subset\left(E_{Y}^{2,0} \oplus E_{Y}^{1,1} \oplus E_{Y}^{0,2}, \theta_{Y}\right)$ denote the unique saturated Higgs subsheaf generated by $E_{Y}^{2,0}$ and $\theta_{Y}$. Then $F_{Y}^{2,0}=E_{Y}^{2,0}$ and $F_{Y}^{0,2}=$ 
$E_{Y}^{0,2}$, since the Griffiths-Yukawa coupling does not vanish. The non-vanishing of the Griffiths-Yukawa coupling along $C_{i}$ implies that

$$
\mathbb{V}_{C_{i}}^{\prime}=\mathbb{V}^{\prime \prime} \oplus \mathbb{U}^{1,1}
$$

where $\mathbb{V}^{\prime \prime}$ is a sub-VHS with rank one Hodge bundles

$$
E_{C_{i}}^{2,0} \oplus E_{C_{i}}^{1,1^{\prime}} \oplus E_{C_{i}}^{2,0}
$$

as above and $U_{C_{i}}^{1,1}$ is a sub-VHS of pure Hodge type $(1,1)$. Using condition (HHP) for $C_{i} \subset Y$ we see that

$$
\begin{aligned}
\left.F_{Y}^{1,1}\right|_{C_{i}} & =\theta_{C_{i}}\left(T_{C_{i}}\left(-\log S_{C_{i}}\right) \otimes E_{C_{i}}^{2,0}\right) \oplus \theta_{N_{C_{i} / Y}}\left(N_{C_{i} / Y} \otimes E_{C_{i}}^{2,0}\right) \\
& =E_{C_{i}}^{1,1^{\prime}} \oplus \theta_{N_{C_{i} / Y}}\left(N_{C_{i} / Y} \otimes E_{C_{i}}^{2,0}\right),
\end{aligned}
$$

where $\theta_{N_{C_{i} / Y}}\left(N_{C_{i} / Y} \otimes E_{C_{i}}^{2,0}\right)$ is a direct factor of $U_{C_{i}}^{1,1}$. In particular $\operatorname{det}\left(F_{Y}^{1,1}\right) \cdot C_{i}=$ 0 . Hence, $\operatorname{det}\left(F_{Y}^{1,1}\right) \cdot C_{z}=0$, where $C_{z}$ is a smooth curve in the family $\cup_{z \in Z} C_{z}$ and meets $S_{Y}$ transversely. Note that $\operatorname{deg} F_{C_{z}}=0$, and by Simpson's polystability for the logarithmic Higgs subsheaf $F_{C_{z}}, \theta_{C_{z}} \subset\left(E_{C_{z}}^{2,0} \oplus E_{C_{z}}^{1,1} \oplus E_{C_{z}}^{0,2}, \theta_{C_{z}}\right)$ we obtain a corresponding sub-VHS $\mathbb{V}_{C_{z}}^{\prime \prime \prime} \subset \mathbb{V}_{C_{z}}^{\prime}$. Since $H\left(Y^{0}\right)$ is $\mathbb{Q}$-simple, the same argument as in the first proof of (b) shows that $\mathbb{V}_{C_{z}}^{\prime \prime \prime}$ extends to a sub-VHS over $Y^{0}$, which uniformizes $Y^{0}$ as a special subvariety of orthogonal type.

(c) The vanishing of the Griffiths-Yukawa coupling on $Y$ implies that the Higgs subsheaf generated by $E_{Y}^{2,0}$ and $\theta_{Y}$ has the form

$$
\left(F_{Y}, \theta_{Y}\right)=\left(E_{Y}^{2,0} \oplus \theta_{Y}\left(T_{Y}\left(-\log S_{Y}\right) \otimes E_{Y}^{2,0}\right), \theta_{Y}\right) .
$$

Therefore one has

$\theta_{Y}\left(T_{Y}\left(-\log S_{Y}\right) \otimes E_{Y}^{2,0}\right) \otimes \mathcal{O}_{C_{i}}=\theta_{C_{i}}\left(T_{C_{i}}\left(-\log S_{C_{i}}\right) \otimes E_{C_{i}}^{2,0}\right) \oplus \theta_{N_{C_{i} / Y}}\left(N_{C_{i} / Y} \otimes E_{C_{i}}^{2,0}\right)$.

Note that in this case

$$
E_{C_{i}}^{2,0}=\mathcal{L}, \quad E_{C_{i}}^{1,1}=\mathcal{L}^{-1} \oplus \mathcal{L} \oplus \mathcal{U}^{1,1},
$$

where $\mathcal{U}^{1,1}$ is polystable of degree zero and such that

$$
\theta_{C_{i}}: T_{C_{i}}\left(-\log S_{C_{i}}\right) \otimes \mathcal{L} \stackrel{\simeq}{\longrightarrow} \mathcal{L}^{-1} \text {, with } \mathcal{L}^{-1}=\theta_{C_{i}}\left(T_{C_{i}}\left(-\log S_{C_{i}}\right) \otimes E_{C_{i}}^{2,0}\right) .
$$

The condition (HHP) for all $C_{i}$ just means that $\theta_{N_{C_{i} / Y}}\left(N_{C_{i} / Y} \otimes E_{C_{i}}^{2,0}\right)$ is a direct factor of $\mathcal{U}^{1,1}$, hence it has degree zero, too. That implies that $\operatorname{det} F_{Y} \cdot C_{i}=0$. By the same argument as in (b) we obtain that $\left(F_{Y}, \theta_{Y}\right)$ corresponds to a sub-VHS over $Y^{0}$, which uniformizes $Y^{0}$ as a special subvariety of unitary type. 


\section{REFERENCES}

[1] A. Ash, D. Mumford, M. Rapoport And Y.-S. TAI: Smooth compactifications of locally symmetric varieties, second edition, Cambridge (2010).

[2] M. АтічAн: On the Krull-Schmidt theorem with application to sheaves, Bull. Soc. Math. France, Vol. 84, 307-317 (1956).

[3] G. BARThel, F. Hirzebruch AND T. HöFER: Geradenkonfigurationen und Algebraische Flächen, Aspects of Math. D4, Friedr. Vieweg \& Sohn, Braunschweig (1987).

[4] R. Borcherds: Automorphic forms with singularities on Grassmannians, Invent. math. 132, 491-562 (1998).

[5] P. Deligne: La conjecture de Weil pour les surfaces K3, Inventiones Math., Vol. 15, 206-226 (1971).

[6] P. Deligne: Variétés de Shimura: interprétation modulaire, et techniques de construction de modèles canoniques, Proceedings of Symposia in Pure Mathematics Vol. 33, part 2, 247-290 (1979).

[7] G. FAltings: Arakelov's theorem on abelian varieties, Inventiones Math., Vol. 73, 337347 (1983).

[8] F. HirzeBruch: Hilbert modular surfaces, L'Ens. Math. 19, 57-113 (1973).

[9] F. Hörmann: The arithmetic volume of Shimura varieties of orthogonal type, dissertation, University of Berlin (2010).

[10] B. Klingler And A. Yafaev: The André-Oort conjecture, preprint (2006).

[11] R. Kоттwitz: Points on some Shimura varieties over finite fields, Journal of the American Mathematical Society, vol.5, No.2, 373-444 (1992).

[12] S. KudLA: Arithmetic cycles on Shimura varieties of orthogonal type, Duke Mathematical Journal, Vol.86, No.1, 39-78 (1997).

[13] M. KUGA AND I SATAKe: Abelian varieties attached to polarized K3-surfaces, Math. Ann. Vol. 169, 239-242 (1967).

[14] J. MiLne: Introduction to Shimura varieties, in: Harmonic analysis, the trace formula, and Shimura varieties, AMS (2005).

[15] M. Möller, E.VIehweg AND K. Zuo: Stability of Hodge bundles and a numerical characterization of Shimura varieties, see arXiv:0706.3462 (2007).

[16] S. Möller, E.Viehweg And K. Zuo: Special families of curves, of Abelian varieties, and of certain minimal manifolds over curves, Global Aspects of Complex Geometry. Springer Verlag, 417-450 (2006).

[17] B. Moonen: Linearity properties of Shimura varieties I, Journal of Alg. Geom., Vol. 7, 539-567 (1998).

[18] B.Moonen Models of Shimura varieties in mixed characteristics, in: Galois representations in arithmetic algebraic geometry, LMS Lecture Notes Series 254, Cambridge University Press, 267-350 (1998).

[19] S. MÜller-Stach, E.VIehweg And K. Zuo: Relative Proportionality for subvarieties of moduli spaces of K3 and abelian surfaces, Pure and Applied Mathematics Quarterly, Vol. 5, Nr. 3, 1161-1199 (2009). 
[20] D. Mumford: Hirzebruch's proportionality theorem in the non-compact case, Inventiones Math., Vol. 42, 239-277 (1977).

[21] M.-H. SAito And S. ZuCKeR: Classification of non-rigid families of K3 surfaces and a finiteness theorem of Arakelov type, Math. Annalen, Vol. 289, 1-31 (1991).

[22] K. SHIH: Existence of certain canonical models, Duke Mathematical Journal, Vol.45, No.1, 63-66 (1978).

[23] C. Simpson: Harmonic bundles on non-compact curves, Journal of the AMS, Vol. 3, 713-770 (1990).

[24] C. Simpson: Higgs bundles and local systems, Publ. Math. IHES vol. 75, 5-95 (1992).

[25] E. VIEhWEg AND K. ZUO: A characterization of certain Shimura curves in the moduli stack of abelian varieties, J. Differential Geometry, Vol. 66, 233-287 (2004).

[26] K. ZUO: Representations of fundamental groups of algebraic varieties, Lecture Notes in Mathematics 1708 Springer (1999).

Stefan Müller-Stach

Universität Mainz, Fachbereich 17, Mathematik, 55099 Mainz, Germany

E-mail: stach@uni-mainz.de

Kang Zuo

Universität Mainz, Fachbereich 17, Mathematik, 55099 Mainz, Germany

E-mail: zuok@uni-mainz.de 